\title{
On the Variational Interpretation of the Discrete KP Equation
}

\author{
Raphael Boll, Matteo Petrera and Yuri B. Suris
}

\begin{abstract}
We study the variational structure of the discrete Kadomtsev-Petviashvili (dKP) equation by means of its pluri-Lagrangian formulation. We consider the dKP equation and its variational formulation on the cubic lattice $\mathbb{Z}^{N}$ as well as on the root lattice $Q\left(A_{N}\right)$. We prove that, on a lattice of dimension at least four, the corresponding Euler-Lagrange equations are equivalent to the $\mathrm{dKP}$ equation.
\end{abstract}

\section{Introduction}

We developed the theory of pluri-Lagrangian problems (integrable systems of variational origin) in recent papers [2-6, 15, 16], influenced by the fundamental insight of $[11-13,17]$. In the present paper, we consider the pluri-Lagrangian formulation of the discrete bilinear Kadomtsev-Petviashvili (dKP) equation on three-dimensional lattices and its consistent extension to higher dimensional lattices. This equation belongs to integrable octahedron-type equations which were classified in [1]. A Lagrangian formulation of this equation was given in [13]. There, the authors consider a discrete 3-form on the lattice $\mathbb{Z}^{3}$ together with the corresponding Euler-Lagrange equations which are shown to be satisfied on solutions of the dKP equation. They also show that this 3-form is closed on solutions of the dKP equation, namely, the so-called $4 \mathrm{D}$ closure relation is satisfied. The main goal of the present paper is to provide a more precise understanding of the findings in that paper. More concretely:

- In the framework of the pluri-Lagrangian formulation, we construct the elementary building blocks of Euler-Lagrange equations, which, in the present situation, are the so-called $4 \mathrm{D}$ corner equations.

R. Boll · M. Petrera $\cdot$ Y.B. Suris $(\bowtie)$

Inst. für Mathematik, Technische Universität Berlin,

Straße des 17. Juni 136, 10623 Berlin, Germany

e-mail: suris@math.tu-berlin.de

R. Boll

e-mail: boll@math.tu-berlin.de

M. Petrera

e-mail: petrera@math.tu-berlin.de

(C) The Author(s) 2016

A.I. Bobenko (ed.), Advances in Discrete Differential Geometry,

DOI 10.1007/978-3-662-50447-5_12 
- In the two-dimensional case, as noticed in [4], the corresponding 3D corner equations build a consistent system. Its solutions are more general then the solutions of the underlying hyperbolic system of quad-equations. On the contrary, in the present three-dimensional situation, the system of 4D corner equations is not consistent in the usual sense (i.e., it does not allow to determine general solutions with the maximal number of initial data). However, this system turns out to be equivalent, in a sense which we are going to explain later, to the corresponding hyperbolic system, namely the dKP equation.

- We provide a rigorous consideration of the branches of the logarithm functions involved in the Euler-Lagrange equations. This leads to the following more precise result: the system of 4D corner equations is equivalent, and thus provides a variational formulation, to two different hyperbolic equations, namely the dKP equation itself and its version obtained under inversion $x \mapsto x^{-1}$ of all fields which will be denoted by $\mathrm{dKP}^{-}$.

One can consider the dKP equation on the cubic lattice $\mathbb{Z}^{3}$ and its higher dimensional analogues $\mathbb{Z}^{N}$, but, as discussed in [1, 8, 9] another natural setting the dKP equation (and related octahedron-type equations) is the three-dimensional root lattice

$$
Q\left(A_{3}\right):=\left\{\left(n_{i}, n_{j}, n_{k}, n_{\ell}\right): n_{i}+n_{j}+n_{k}+n_{\ell}=0\right\} .
$$

Also in this setting, the dKP equation can be extended in a consistent way to the higher dimensional lattices $Q\left(A_{N}\right)$ with $N>3$.

Both lattices have their advantages and disadvantages. The cubic lattice $\mathbb{Z}^{N}$, on the one hand, is more manageable and easier to visualize. Its cell structure is very simple: for every dimension $N$, all $N$-dimensional elementary cells are $N$-dimensional cubes. On the other hand, it is less natural to consider $\mathrm{dKP}$ on the lattice $\mathbb{Z}^{3}$, because this equation depends on the variables assigned to six out of eight vertices of a (threedimensional) cube.

The root lattice $Q\left(A_{N}\right)$, in contrast, has a more complicated cell structure, because the number of different $N$-dimensional elementary cells increases with the dimension $N$. For instance, for $N=3$ there are two types of elementary cells octahedra and tetrahedra. Moreover, especially in higher dimensions, a visualization of the elementary cells is difficult, if not impossible. However, this lattice is more natural for the consideration of $\mathrm{dKP}$ from the combinatorial point of view, because this equation depends on variables which can be assigned to the six vertices of an octahedron, one of the elementary cells of the lattice. Furthermore, the four-dimensional elementary cells are combinatorially smaller (they contain only 10 vertices, as compared with 16 vertices of a four-dimensional cube) and possess higher symmetry than the cubic ones. Since they support the equations which serve as variational analogue of the dKP equation, this leads to a simpler situation.

We will see that a four-dimensional cube is combinatorially equivalent to the sum of four elementary cells of the root lattice $Q\left(A_{4}\right)$. Therefore, several results in the cubic case can be seen as direct consequences of results of the more fundamental $Q\left(A_{N}\right)$-case. 
Let us start with some concrete definitions valid for an arbitrary $N$-dimensional lattice $\mathscr{X}$.

Definition 1.1 (Discrete 3-form) A discrete 3-form on $\mathscr{X}$ is a real-valued function $\mathscr{L}$ of oriented 3 -cells $\sigma$ depending on some field $x: \mathscr{X} \rightarrow \mathbb{R}$, such that $\mathscr{L}$ changes the sign by changing the orientation of $\sigma$.

For instance, in $Q\left(A_{N}\right)$, the 3-cells are tetrahedra and octahedra, and, in $\mathbb{Z}^{N}$, the 3 -cells are $3 \mathrm{D}$ cubes.

Definition 1.2 (3-dimensional pluri-Lagrangian problem) Let $\mathscr{L}$ be a discrete 3form on $\mathscr{X}$ depending on $x: \mathscr{X} \rightarrow \mathbb{R}$.

- To an arbitrary 3-manifold $\Sigma \subset \mathscr{X}$, i.e., a union of oriented 3-cells which forms an oriented three-dimensional topological manifold, there corresponds the action functional, which assigns to $\left.x\right|_{V(\Sigma)}$, i.e., to the fields in the set of the vertices $V(\Sigma)$ of $\Sigma$, the number

$$
S_{\Sigma}:=\sum_{\sigma \in \Sigma} \mathscr{L}(\sigma)
$$

- We say that the field $x: V(\Sigma) \rightarrow \mathbb{R}$ is a critical point of $S_{\Sigma}$, if at any interior point $n \in V(\Sigma)$, we have

$$
\frac{\partial S_{\Sigma}}{\partial x(n)}=0 .
$$

Equation (1) are called discrete Euler-Lagrange equations for the action $S_{\Sigma}$.

- We say that the field $x: \mathscr{X} \rightarrow \mathbb{R}$ solves the pluri-Lagrangian problem for the Lagrangian 3-form $\mathscr{L}$ if, for any 3-manifold $\Sigma \subset \mathscr{X}$, the restriction $\left.x\right|_{V(\Sigma)}$ is a critical point of the corresponding action $S_{\Sigma}$.

In the present paper, we focus on the variational formulation of the $\mathrm{dKP}$ equation on $Q\left(A_{N}\right)$ and $\mathbb{Z}^{N}$. Let us formulate the main results of the paper.

On the lattice $Q\left(A_{N}\right)$, we consider discrete 3-forms vanishing on all tetrahedra. One can show (see Corollary 2.5) that, for an arbitrary interior vertex of any 3-manifold in $Q\left(A_{N}\right)$, the Euler-Lagrange equations follow from certain elementary building blocks. These so-called 4D corner equations are the Euler-Lagrange equations for elementary 4-cells of $Q\left(A_{N}\right)$ different from 4-simplices, so-called 4-ambo-simplices. Such a 4-ambo-simplex has ten vertices. Therefore, the crucial issue is the study of the system consisting of the corresponding ten corner equations. In our case, each corner equation depends on all ten fields at the vertices of the 4ambo-simplex. Therefore, one could call this system consistent if any two equations are functionally dependent. It turns out that this is not the case. We will prove the following statement:

Theorem 1.3 Every solution of the system of ten corner equations for a 4-ambosimplex in $Q\left(A_{N}\right)$ satisfies either the system of five $d K P$ equations or the system of five $d K P^{-}$equations on the five octahedral facets of the 4-ambo-simplex. 
Thus, one can prescribe arbitrary initial values at seven vertices of a 4-ambo-simplex. We will also prove the following theorem:

Theorem 1.4 The discrete 3 -form $\mathscr{L}$ is closed on any solution of the system of corner equations.

In $[4,15]$, it was shown that in dimensions 1 and 2 the analogues of the property formulated in Theorem 1.4 are related to more traditional integrability attributes.

For the case of the cubic lattice $\mathbb{Z}^{N}$, the situation is similar: one can show (see Corollary 4.2) that, for an arbitrary interior vertex of any 3-manifold in $\mathbb{Z}^{3}$, the EulerLagrange equations follow from certain elementary building blocks. These so-called 4D corner equations are the Euler-Lagrange equations for elementary 4D cubes in $\mathbb{Z}^{N}$. A 4D cube has sixteen vertices, but in our case the action on a $4 \mathrm{D}$ cube turns out to be independent of the fields on two of the vertices. Therefore, the crucial issue is the study of the system consisting of the corresponding fourteen corner equations. Six of the fourteen corner equations depend each on thirteen of the fourteen fields. There do not exist pairs of such equations which are independent of one and the same field. All other equations depend each on ten of the fourteen fields. Therefore, one could call this system consistent if it would have the minimal possible rank 2 (assign twelve fields arbitrarily and use two of the six corner equations-depending on thirteen fields - to determine the remaining two fields, then all twelve remaining equations should be satisfied automatically). It turns out that the system of the fourteen corner equations is not consistent in this sense. We will prove the following analogue of Theorem 1.3:

Theorem 1.5 Every solution of the system of fourteen corner equations for a $4 D$ cube in $\mathbb{Z}^{N}$ satisfies either the system of eight dKP equations or the system of eight $d K P^{-}$equations on the eight cubic facets of the $4 D$ cube.

Thus, one can prescribe arbitrary initial values at nine vertices of a 4D cube. Correspondingly, we will also prove the following statement:

Theorem 1.6 The discrete 3-form $\mathscr{L}$ is closed on any solution of the system of corner equations.

The paper is organized as follows: we start with the root lattice $Q\left(A_{N}\right)$, thus considering the combinatorial issues and some general properties of pluri-Lagrangian systems. Then we introduce the dKP equation and its pluri-Lagrangian structure. In the second part of the paper the present similar considerations for the cubic lattice $\mathbb{Z}^{N}$.

\section{The Root Lattice $Q\left(A_{N}\right)$}

We consider the root lattice

$$
Q\left(A_{N}\right):=\left\{n:=\left(n_{0}, n_{1}, \ldots, n_{N}\right) \in \mathbb{Z}^{N+1}: n_{0}+n_{1}+\ldots+n_{N}=0\right\},
$$


where $N \geq 3$. The three-dimensional sub-lattices $Q\left(A_{3}\right)$ are given by

$$
Q\left(A_{3}\right):=\left\{\left(n_{i}, n_{j}, n_{k}, n_{\ell}\right): n_{i}+n_{j}+n_{k}+n_{\ell}=\text { const }\right\} .
$$

We consider fields $x: Q\left(A_{N}\right) \rightarrow \mathbb{R}$, and use the shorthand notations

$$
x_{\bar{\imath}}=x\left(n-e_{i}\right), \quad x=x(n), \quad \text { and } \quad x_{i}=x\left(n+e_{i}\right),
$$

where $e_{i}$ is the unit vector in the $i$ th coordinate direction. Furthermore, the shift functions $T_{i}$ and $T_{\bar{l}}$ are defined by

$$
T_{i} x_{\alpha}:=x_{i \alpha} \text { and } T_{\bar{l}} x_{\alpha}:=x_{\bar{\imath} \alpha}
$$

for a multiindex $\alpha$. For simplicity, we sometimes abuse notations by identifying lattice points $n$ with the corresponding fields $x(n)$.

We now give a very brief introduction to the Delaunay cell structure of the $n$ dimensional root lattice $Q\left(A_{N}\right)[7,14]$. Here, we restrict ourselves to a very elementary description which is appropriate to our purposes and follow the considerations in [1]. For each $N$ there are $N$ sorts of $N$-cells of $Q\left(A_{N}\right)$ denoted by $P(k, N)$ with $k=1, \ldots, N$ :

- Two sorts of 2-cells:

$P(1,2)$ : black triangles $\lfloor i j k\rfloor:=\left\{x_{i}, x_{j}, x_{k}\right\}$;

$P(2,2)$ : white triangles $\lceil i j k\rceil:=\left\{x_{i j}, x_{i k}, x_{j k}\right\}$;

- Three sorts of 3-cells:

$P(1,3)$ : black tetrahedra $\lfloor i j k \ell\rfloor:=\left\{x_{i}, x_{j}, x_{k}, x_{\ell}\right\}$;

$P(2,3):$ octahedra $[i j k \ell]:=\left\{x_{i j}, x_{i k}, x_{i \ell}, x_{j k}, x_{j \ell}, x_{k \ell}\right\}$;

$P(3,3)$ : white tetrahedra $\lceil i j k \ell\rceil:=\left\{x_{i j k}, x_{i j \ell}, x_{i k \ell}, x_{j k \ell}\right\}$;

- Four sorts of 4-cells:

$P(1,4)$ : black 4-simplices $\llbracket i j k \ell m \rrbracket:=\left\{x_{i}, x_{j}, x_{k}, x_{\ell}, x_{m}\right\}$;

$P(2,4)$ : black 4-ambo-simplices $\lfloor i j k \ell m\rfloor:=\left\{x_{\alpha \beta}: \alpha, \beta \in\{i, j, k, \ell, m\}, \alpha \neq \beta\right\}$;

$P(3,4)$ : white 4-ambo-simplices $\lceil i j k \ell m\rceil:=\left\{x_{\alpha \beta \gamma}: \alpha, \beta, \gamma \in\{i, j, k, \ell, m\}\right.$, $\alpha \neq \beta \neq \gamma \neq \alpha\}$

$P(4,4):$ white 4-simplices $\llbracket i j k \ell m \rrbracket:=\left\{x_{i j k \ell}, x_{i j k m}, x_{i j \ell m}, x_{i k \ell m}, x_{j k \ell m}\right\}$.

The facets of 3-cells and 4-cells can be found in Appendix 1.

In the present paper we will consider objects on oriented manifolds. We say that a black triangle $\lfloor i j k\rfloor$ and white triangle $\lceil i j k\rceil$ are positively oriented if $i<j<k$ (see Fig. 1). Any permutation of two indices changes the orientation to the opposite one.

When we use the bracket notation, we always write the letters in brackets in increasing order, so, e.g., in writing $\lfloor i j k\rfloor$ we assume that $i<j<k$ and avoid the notation $\lfloor j i k\rfloor$ or $\lfloor i k j\rfloor$ for the negatively oriented triangle $-\lfloor i j k\rfloor$.

There is a simple recipe to derive the orientation of facets of an $N$-cell: On every index in the brackets we put alternately a "+" or a "-" starting with a "+" on the 
Fig. 1 Orientation of triangles: a the black triangle $\lfloor i j k\rfloor ; \mathbf{b}$ the white triangle $\lceil i j k\rceil$
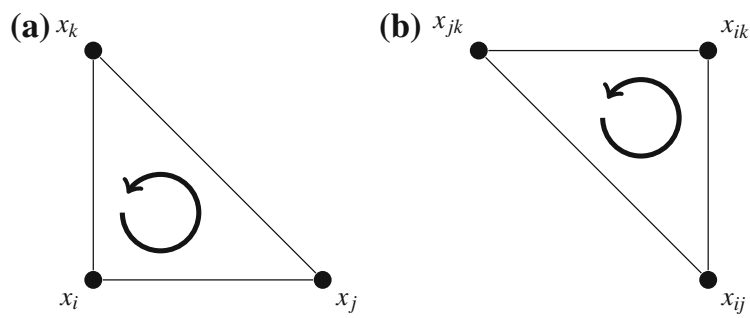

last index. Then we get each of its facets by deleting one index and putting the corresponding sign in front of the bracket. For instance, the black 4-ambo-simplex

$$
\left[\begin{array}{ccccc}
+ & - & + & - \\
i & j & k & \ell & m
\end{array}\right\rfloor
$$

has the five octahedral facets $[i j k \ell],-[i j k m],[i j \ell m],-[i k \ell m]$, and $[j k \ell m]$.

The following two definitions are valid for arbitrary $N$-dimensional lattices $\mathscr{X}$.

Definition 2.1 (Adjacent $N$-cell) Given an $N$-cell $\sigma$, another $N$-cell $\bar{\sigma}$ is called adjacent to $\sigma$ if $\sigma$ and $\bar{\sigma}$ share a common $(N-1)$-cell. The orientation of this $(N-1)$-cell in $\sigma$ must be opposite to its orientation in $\bar{\sigma}$.

The latter property guarantees that the orientations of the adjacent $N$-cells agree.

Definition 2.2 (Flower) A 3-manifold in $\mathscr{X}$ with exactly one interior vertex $x$ is called a flower with center $x$. The flower at an interior vertex $x$ of a given 3-manifold is the flower with center $x$ which lies completely in the 3-manifold.

As a consequence, in $Q\left(A_{N}\right)$, in each flower every tetrahedron has exactly three adjacent 3-cells and every octahedron has exactly four adjacent 3-cells.

Examples for open 3-manifolds in $Q\left(A_{N}\right)$ are the three-dimensional sub-lattices $Q\left(A_{3}\right)$. Here, the flower at an interior vertex consists of eight tetrahedra (four black and four white ones) and six octahedra.

Examples of closed 3-manifolds in $Q\left(A_{N}\right)$ are the set of facets of a 4-ambosimplex (consisting of five tetrahedra) and the set of facets of a 4-ambo-simplex (consisting of five tetrahedra and five octahedra).

The elementary building blocks of 3-manifolds are so-called 4D corners:

Definition 2.3 (4D corner) A $4 D$ corner with center $x$ is a 3-manifold consisting of all facets of a 4-cell adjacent to $x$.

In $Q\left(A_{N}\right)$, there are two different types of 4D corners: a corner on a 4-simplex (consisting of a four tetrahedra) and a corner on a 4-ambo-simplex (consisting of two tetrahedra and three octahedra), see Appendix 2 for details.

The following combinatorial statement will be proven in Appendix 3:

Theorem 2.4 The flower at any interior vertex of any 3-manifold in $Q\left(A_{N}\right)$ can be represented as a sum of $4 D$ corners in $Q\left(A_{N+2}\right)$. 
Let $\mathscr{L}$ be a discrete 3 -form on $Q\left(A_{N}\right)$. The exterior derivative $d \mathscr{L}$ is a discrete 4-form whose value at any 4-cell in $Q\left(A_{N}\right)$ is the action functional of $\mathscr{L}$ on the 3 manifold consisting of the facets of the 4-cell. For our purposes, we consider discrete 3 -forms $\mathscr{L}$ vanishing on all tetrahedra. In particular, we have

$$
d \mathscr{L}(\llbracket i j k \ell m \rrbracket) \equiv 0 \text { and } d \mathscr{L}(\llbracket i j k \ell m \rrbracket) \equiv 0
$$

since a 4-simplices only contain tetrahedra. The exterior derivative on a black 4ambo-simplex $\lfloor i j k \ell m\rfloor$ is given by

$$
\begin{aligned}
\underline{S}^{i j k \ell m}: & =d \mathscr{L}(\lfloor i j k \ell m\rfloor) \\
& =\mathscr{L}([i j k \ell])+\mathscr{L}(-[i j k m])+\mathscr{L}([i j \ell m])+\mathscr{L}(-[i k \ell m])+\mathscr{L}([j k \ell m]) .
\end{aligned}
$$

The exterior derivative on a white 4-ambo-simplex $\lceil i j k \ell m\rceil$ is given by

$$
\begin{aligned}
\bar{S}^{i j k \ell m}:= & d \mathscr{L}(\lceil i j k \ell m\rceil) \\
= & \mathscr{L}\left(T_{m}[i j k \ell]\right)+\mathscr{L}\left(-T_{\ell}[i j k m]\right)+\mathscr{L}\left(T_{k}[i j \ell m]\right)+\mathscr{L}\left(-T_{j}[i k \ell m]\right) \\
& +\mathscr{L}\left(T_{i}[j k \ell m]\right) .
\end{aligned}
$$

Accordingly, the Euler-Lagrange equations on black 4-ambo-simplices $\lfloor i j k \ell m\rfloor$ are

$$
\begin{aligned}
& \frac{\partial \underline{S}^{i j k \ell m}}{\partial x_{i j}}=0, \quad \frac{\partial \underline{S}^{i j k \ell m}}{\partial x_{i k}}=0, \quad \frac{\partial \underline{S}^{i j k \ell m}}{\partial x_{i \ell}}=0, \quad \frac{\partial \underline{S}^{i j k \ell m}}{\partial x_{i m}}=0, \quad \frac{\partial \underline{S}^{i j k \ell m}}{\partial x_{j k}}=0, \\
& \frac{\partial \underline{S}^{i j k \ell m}}{\partial x_{j \ell}}=0, \quad \frac{\partial \underline{S}^{i j k \ell m}}{\partial x_{j m}}=0, \quad \frac{\partial \underline{S}^{i j k \ell m}}{\partial x_{k \ell}}=0, \quad \frac{\partial \underline{S}^{i j k \ell m}}{\partial x_{k m}}=0, \quad \frac{\partial \underline{S}^{i j k \ell m}}{\partial x_{\ell m}}=0 .
\end{aligned}
$$

and the Euler-Lagrange equations on white 4-ambo-simplices $\lceil i j k \ell m\rceil$ are

$$
\begin{aligned}
& \frac{\partial \bar{S}^{i j k \ell m}}{\partial x_{i j k}}=0, \quad \frac{\partial \bar{S}^{i j k \ell m}}{\partial x_{i j \ell}}=0, \quad \frac{\partial \bar{S}^{i j k \ell m}}{\partial x_{i j m}}=0, \quad \frac{\partial \bar{S}^{i j k \ell m}}{\partial x_{i k \ell}}=0, \quad \frac{\partial \bar{S}^{i j k \ell m}}{\partial x_{i k m}}=0, \\
& \frac{\partial \bar{S}^{i j k \ell m}}{\partial x_{i \ell m}}=0, \quad \frac{\partial \bar{S}^{i j k \ell m}}{\partial x_{j k \ell}}=0, \quad \frac{\partial \bar{S}^{i j k \ell m}}{\partial x_{j k m}}=0, \quad \frac{\partial \bar{S}^{i j k \ell m}}{\partial x_{j \ell m}}=0, \quad \frac{\partial \bar{S}^{i j k \ell m}}{\partial x_{k \ell m}}=0 .
\end{aligned}
$$

The last two systems are called corner equations.

The following statement is an immediate consequence of Theorem 2.4:

Theorem 2.5 For discrete every 3-form on $Q\left(A_{N}\right)$ and every 3-manifold in $Q\left(A_{N}\right)$ all corresponding Euler-Lagrange equations can be written as a sum of corner equations. 


\section{The dKP Equation on $Q\left(A_{N}\right)$}

We will now introduce the dKP equation on the root lattice $Q\left(A_{3}\right)$. Every oriented octahedron [ijkl] $(i<j<k<\ell)$ in $Q\left(A_{3}\right)$ supports the equation

$$
x_{i j} x_{k \ell}-x_{i k} x_{j \ell}+x_{i \ell} x_{j k}=0 .
$$

We can extend this system in a consistent way (see [1]) to the four-dimensional root lattice $Q\left(A_{4}\right)$ and higher-dimensional analogues, such that the five octahedral facets $[i j k \ell],[j k \ell m],-[i k \ell m],[i j m \ell]$, and $-[i j k m]$ of the black 4-ambo-simplex $\lfloor i j k \ell m\rfloor$ support the equations

$$
\begin{aligned}
& x_{i j} x_{k \ell}-x_{i k} x_{j \ell}+x_{i \ell} x_{j k}=0, \\
& x_{j k} x_{\ell m}-x_{j \ell} x_{k m}+x_{j m} x_{k \ell}=0, \\
& x_{k \ell} x_{i m}-x_{k m} x_{i \ell}+x_{i k} x_{\ell m}=0, \\
& x_{\ell m} x_{i j}-x_{i \ell} x_{j m}+x_{j \ell} x_{i m}=0, \\
& x_{i m} x_{j k}-x_{j m} x_{i k}+x_{k m} x_{i j}=0
\end{aligned}
$$

and the five octahedral facets $T_{m}[i j k \ell], T_{i}[j k \ell m],-T_{j}[i k \ell m], T_{k}[i j \ell m]$, and $-T_{\ell}[i j \mathrm{~km}]$ of the white 4-ambo-simplex $\lceil i j k \ell m\rceil$ support the equations

$$
\begin{aligned}
& x_{i j m} x_{k \ell m}-x_{i k m} x_{j \ell m}+x_{i \ell m} x_{j k m}=0, \\
& x_{i j k} x_{i \ell m}-x_{i j \ell} x_{i k m}+x_{i j m} x_{i k \ell}=0, \\
& x_{j k \ell} x_{i j m}-x_{j k m} x_{i j \ell}+x_{i j k} x_{j \ell m}=0, \\
& x_{k \ell m} x_{i j k}-x_{i k \ell} x_{j k m}+x_{j k \ell} x_{i k m}=0, \\
& x_{i \ell m} x_{j k \ell}-x_{j \ell m} x_{i k \ell}+x_{k \ell m} x_{i j \ell}=0 .
\end{aligned}
$$

In both systems one can derive one equation from another by cyclic permutations of indices $(i j k \ell m)$.

We propose the following discrete 3 -form $\mathscr{L}$ defined on oriented octahedra $[i j k \ell]$ :

$$
\mathscr{L}([i j k \ell]):=\frac{1}{2}\left(\Lambda\left(\frac{x_{i j} x_{k \ell}}{x_{i k} x_{j \ell}}\right)+\Lambda\left(\frac{x_{i k} x_{j \ell}}{x_{i \ell} x_{j k}}\right)+\Lambda\left(-\frac{x_{i \ell} x_{j k}}{x_{i j} x_{k \ell}}\right)\right),
$$

where

$$
\Lambda(z):=\lambda(z)-\lambda\left(\frac{1}{z}\right) \text { and } \lambda(z):=-\int_{0}^{z} \frac{\log |1-x|}{x} d x .
$$

The discrete 3-form (9) has its motivation in [13]. Indeed, in [13], the authors consider a similar discrete 3 -form on the cubic lattice $\mathbb{Z}^{N}$. One can also consider our 3-form on the cubic lattice $\mathbb{Z}^{N}$. Then one would assign to each $3 \mathrm{D}$ cube the 3 -form at its 
inscribed octahedron. This 3-form differs from their one by an additive constant and a slightly different definition of the function $\lambda(z)$ : they use the function

$$
\operatorname{Li}_{2}(z):=-\int_{0}^{z} \frac{\log (1-x)}{x} d x
$$

instead of $\lambda(z)$. Our choice of $\lambda(z)$ allows us for a more precise consideration of the branches of the occurring logarithm.

Observe that the expression (9) only changes its sign under the cyclic permutation of indices $(i j k \ell m)$. This follows from $\Lambda(z)=-\Lambda\left(z^{-1}\right)$. As a consequence, the exterior derivatives $\underline{S}^{i j k \ell m}$ and $\bar{S}^{i j k \ell m}$ defined in (2) and (3), respectively, are invariant under the cyclic permutation of indices $(i j k \ell m)$. Therefore, one can obtain all corner equations in (4) and (5) by (iterated) cyclic permutation ( $i j k \ell m)$ from

$$
\frac{\partial \underline{S}^{i j k \ell m}}{\partial x_{i j}}=0, \quad \frac{\partial \underline{S}^{i j k \ell m}}{\partial x_{i k}}=0, \quad \text { and } \quad \frac{\partial \bar{S}^{i j k \ell m}}{\partial x_{i j k}}=0, \quad \frac{\partial \bar{S}^{i j k \ell m}}{\partial x_{i j \ell}}=0 .
$$

Let us study separately the corner equations on black and white 4-ambo-simplices. The corner equations which live on the black 4-ambo-simplex $\lfloor i j k \ell m\rfloor$ are given by

$$
\frac{\partial \underline{S}^{i j k \ell m}}{\partial x_{i j}}=\frac{\partial \mathscr{L}([i j k \ell])}{\partial x_{i j}}+\frac{\partial \mathscr{L}(-[i j k m])}{\partial x_{i j}}+\frac{\partial \mathscr{L}([i j \ell m])}{\partial x_{i j}}=0
$$

and

$$
\frac{\partial \underline{S}^{i j k \ell m}}{\partial x_{i k}}=\frac{\partial \mathscr{L}([i j k \ell])}{\partial x_{i k}}+\frac{\partial \mathscr{L}(-[i j k m])}{\partial x_{i k}}+\frac{\partial \mathscr{L}(-[i k \ell m])}{\partial x_{i k}}=0 .
$$

Explicitly, they read

$$
\frac{1}{x_{i j}} \log \left|E_{i j}\right|=0 \text { and } \frac{1}{x_{i k}} \log \left|E_{i k}\right|=0,
$$

where

$$
E_{i j}:=\frac{x_{i j} x_{k \ell}+x_{i \ell} x_{j k}}{x_{i j} x_{k \ell}-x_{i k} x_{j \ell}} \cdot \frac{x_{i j} x_{k m}-x_{i k} x_{j m}}{x_{i j} x_{k m}+x_{i m} x_{j k}} \cdot \frac{x_{i j} x_{\ell m}+x_{i m} x_{j \ell}}{x_{i j} x_{\ell m}-x_{i \ell} x_{j m}}
$$

and

$$
E_{i k}:=\frac{x_{i k} x_{j \ell}-x_{i j} x_{k \ell}}{x_{i k} x_{j \ell}-x_{i \ell} x_{j k}} \cdot \frac{x_{i k} x_{j m}-x_{i m} x_{j k}}{x_{i k} x_{j m}-x_{i j} x_{k m}} \cdot \frac{x_{i k} x_{\ell m}-x_{i \ell} x_{k m}}{x_{i k} x_{\ell m}+x_{i m} x_{k \ell}}
$$

For every corner equation (12) there are two classes of solutions, because any solution can either solve $E_{i j}=-1$ or $E_{i j}=1$. Hereafter, we only consider solutions, where all fields $x_{i j}$ are non-zero (we call such solutions non-singular). 
Theorem 3.1 Every solution of the system (4) solves either the system

$$
\begin{gathered}
E_{i j}=-1, \quad E_{i k}=-1, \quad E_{i \ell}=-1, \quad E_{i m}=-1, \quad E_{j k}=-1, \\
E_{j \ell}=-1, \quad E_{j m}=-1, \quad E_{k \ell}=-1, \quad E_{k m}=-1, \quad E_{\ell m}=-1
\end{gathered}
$$

or the system

$$
\begin{aligned}
& E_{i j}=1, \quad E_{i k}=1, \quad E_{i \ell}=1, \quad E_{i m}=1, \quad E_{j k}=1, \\
& E_{j \ell}=1, \quad E_{j m}=1, \quad E_{k \ell}=1, \quad E_{k m}=1, \quad E_{\ell m}=1 .
\end{aligned}
$$

Furthermore, the system (13) is equivalent to the system (7) (that is dKP on the corresponding black 4-ambo-simplex). The system (14) is equivalent to the system

$$
\begin{aligned}
& x_{i k} x_{i \ell} x_{j k} x_{j \ell}-x_{i j} x_{i \ell} x_{j k} x_{k \ell}+x_{i j} x_{i k} x_{j \ell} x_{k \ell}=0, \\
& x_{j \ell} x_{j m} x_{k \ell} x_{k m}-x_{j k} x_{j m} x_{k \ell} x_{\ell m}+x_{j k} x_{j \ell} x_{k m} x_{\ell m}=0, \\
& x_{k m} x_{i k} x_{\ell m} x_{i \ell}-x_{k \ell} x_{i k} x_{\ell m} x_{i m}+x_{k \ell} x_{k m} x_{i \ell} x_{i m}=0, \\
& x_{i \ell} x_{j \ell} x_{i m} x_{j m}-x_{\ell m} x_{j \ell} x_{i m} x_{i j}+x_{\ell m} x_{i \ell} x_{j m} x_{i j}=0, \\
& x_{j m} x_{k m} x_{i j} x_{i k}-x_{i m} x_{k m} x_{i j} x_{j k}+x_{i m} x_{j m} x_{i k} x_{j k}=0,
\end{aligned}
$$

which is the system (7) after the transformation $x \mapsto x^{-1}$ of fields (that is dKP $P^{-}$on the corresponding black 4-ambo-simplex).

Proof Consider a solution $x$ of (4) that solves $E_{i j}=-1$ and $E_{j k}=-1$. We set

$$
\begin{aligned}
& a_{i j}:=x_{\ell m} x_{i j}-x_{i \ell} x_{j m}+x_{j \ell} x_{i m}, \\
& a_{i k}:=x_{k \ell} x_{i m}-x_{k m} x_{i \ell}+x_{i k} x_{\ell m},
\end{aligned}
$$

and

$$
a_{j k}:=x_{j k} x_{\ell m}-x_{j \ell} x_{k m}+x_{j m} x_{k \ell}
$$

and use these equations to substitute $x_{i j}, x_{i k}$ and $x_{j k}$ in $E_{i j}=-1$ and $E_{j k}=-1$. Writing down the result in polynomial form, we get

$$
x_{\ell m}^{2}\left(a_{i j}+x_{i \ell} x_{j m}-x_{i m} x_{j \ell}\right) e_{i j}=0
$$

and

$$
x_{\ell m}^{2}\left(a_{j k}+x_{j \ell} x_{k m}-x_{j m} x_{k \ell}\right) e_{j k}=0,
$$

where $e_{i j}$ and $e_{j k}$ are certain polynomials. Since for every solutions of (4) all fields are non-zero this leads us to $e_{i j}=0$ and $e_{j k}=0$. Computing the difference of the latter two equations we get 


$$
a_{i j} x_{k \ell} x_{k m}\left(a_{i j}+x_{i \ell} x_{j m}-x_{i m} x_{j \ell}\right)-a_{j k} x_{i \ell} x_{i m}\left(a_{j k}+x_{j \ell} x_{k m}-x_{j m} x_{k \ell}\right)=0
$$

and, with the use of (16) and (18),

$$
x_{\ell m}\left(a_{i j} x_{i j} x_{k \ell} x_{k m}-a_{j k} x_{j k} x_{i \ell} x_{i m}\right)=0,
$$

which depends on seven independent fields, i.e., no subset of six fields belong to one octahedron. Then comparing coefficients leads to $a_{i j}=a_{j k}=0$. Substituting

$$
x_{i j}=\frac{x_{i \ell} x_{j m}-x_{i m} x_{j \ell}}{x_{\ell m}} \text { and } x_{j k}=\frac{x_{j \ell} x_{k m}-x_{j m} x_{k \ell}}{x_{\ell m}}
$$

into $E_{i j}=-1$ and solving the resulting equation with respect to $x_{i k}$, we get

$$
x_{i k}=\frac{x_{i \ell} x_{k m}-x_{i m} x_{k \ell}}{x_{\ell m}} .
$$

Substituting $x_{i j}, x_{i k}$ and $x_{j k}$ in $E_{i k}$ by using the last three equations, we get $E_{i k}=-1$.

Analogously, one can prove that, for a solution $x$ of (4) which solves $E_{i j}=-1$ and $E_{i k}=-1$, we have $E_{j k}=-1$, and for a solution $x$ of (4) which solves $E_{i k}=-1$ and $E_{i \ell}=-1$, we have $E_{k \ell}=-1$. Therefore, for every solution $x$ of (4) and for every white triangle $\left\{x_{\alpha}, x_{\beta}, x_{\gamma}\right\}$ on the black 4-ambo-simplex $\lfloor i j k \ell m\rfloor$ we proved the following: if $E_{\alpha}=-1$ and $E_{\beta}=-1$ then $E_{\gamma}=-1$, too.

On the other hand, one can easily see that $x$ solves $E_{i j}=1$ or $E_{j k}=1$ if and only if $x^{-1}$ solves $E_{i j}=-1$ or $E_{i k}=-1$, respectively. Therefore, we also know that, if $E_{\alpha}=1$ and $E_{\beta}=1$ then $E_{\gamma}=1$, too.

Summarizing, we proved that every solution $x$ of (4) solves either (13) and then also (7) or (14) and then also (15).

Consider a non-singular solution $x$ of the system (7). Then

$$
\begin{aligned}
E_{i j} & =\frac{x_{i j} x_{k \ell}+x_{i \ell} x_{j k}}{x_{i j} x_{k \ell}-x_{i k} x_{j \ell}} \cdot \frac{x_{i j} x_{k m}-x_{i k} x_{j m}}{x_{i j} x_{k m}+x_{i m} x_{j k}} \cdot \frac{x_{i j} x_{\ell m}+x_{i m} x_{j \ell}}{x_{i j} x_{\ell m}-x_{i \ell} x_{j m}} \\
& =\frac{x_{i k} x_{j \ell}}{-x_{i \ell} x_{j k}} \cdot \frac{-x_{i m} x_{j k}}{x_{i k} x_{j m}} \cdot \frac{x_{i \ell} x_{j m}}{-x_{i m} x_{j \ell}}=-1
\end{aligned}
$$

and

$$
\begin{aligned}
E_{i k} & =\frac{x_{i k} x_{j \ell}-x_{i j} x_{k \ell}}{x_{i k} x_{j \ell}-x_{i \ell} x_{j k}} \cdot \frac{x_{i k} x_{j m}-x_{i m} x_{j k}}{x_{i k} x_{j m}-x_{i j} x_{k m}} \cdot \frac{x_{i k} x_{\ell m}-x_{i \ell} x_{k m}}{x_{i k} x_{\ell m}+x_{i m} x_{k \ell}} \\
& =\frac{x_{i \ell} x_{j k}}{x_{i j} x_{k \ell}} \cdot \frac{x_{i j} x_{k m}}{x_{i m} x_{j k}} \cdot \frac{x_{i m} x_{k \ell}}{-x_{i \ell} x_{k m}}=-1 .
\end{aligned}
$$

This proves the equivalence of (13) and (7) and also the equivalence of (14) and (15) since $x$ solves $E_{i j}=-1$ or (7) if and only if $x^{-1}$ solves $E_{i j}=1$ or (15), respectively. 
We will present the closure relation which can be seen as a criterion of integrability:

Theorem 3.2 (Closure relation) There holds:

$$
\underline{S}^{i j k \ell m} \pm \frac{\pi^{2}}{4}=0
$$

on all solutions of (13) and (14), respectively. Therefore, one can redefine the 3-form $\mathscr{L}$ as

$$
\tilde{\mathscr{L}}([i j k \ell]):=\mathscr{L}([i j k \ell]) \pm \frac{\pi^{2}}{4}
$$

in order to get $\underline{S}^{i j k \ell m}=0$ on all solutions of (13) and (14), respectively.

Proof The set of solutions $\mathscr{S}^{+}$of (13), as well as the set of solutions $\mathscr{S}^{-}$(14), is a connected seven-dimensional algebraic manifold which can be parametrized by the set of variables $\left\{x_{i j}, x_{i k}, x_{i \ell}, x_{i m}, x_{j k}, x_{j \ell}, x_{j m}\right\}$. We want to show that the directional derivatives of $\underline{S}^{i j k \ell m}$ along tangent vectors of $\mathscr{S}^{ \pm}$vanish. It is easy to see that the stronger property $\operatorname{grad} \underline{S}^{i j k \ell m}=0$ on $\mathscr{S}^{ \pm}$, where we $\underline{S}^{i j k \ell m}$ is considered as a function of ten variables $x_{i j}$, is a consequence of (13), respectively (14). Therefore, the function $\underline{S}^{i j k \ell m}$ is constant on $\mathscr{S}^{ \pm}$.

To determine the value of $\underline{S}^{i j k \ell m}$ on solutions of (13), we consider the constant solution of (7)

$$
\begin{aligned}
& x_{i j}=x_{j k}=x_{k \ell}=x_{\ell m}=x_{i m}=a, \\
& x_{i k}=x_{j \ell}=x_{k m}=x_{i \ell}=x_{j m}=-1,
\end{aligned}
$$

where

$$
a:=\frac{1}{2}-\frac{\sqrt{5}}{2} .
$$

(Indeed, for this point every equation from (7) looks like $a^{2}-1-a=0$.) Therefore, this point satisfies (13), because (7) and (13) are equivalent.

Consider the dilogarithm as defined in (11) and suppose that $z>1$. According to [10], we derive:

$$
\operatorname{Li}_{2}(z)=-\mathrm{Li}_{2}\left(z^{-1}\right)-\frac{1}{2} \log ^{2} z+\frac{\pi^{2}}{3}-i \pi \log z
$$

and

$$
\begin{aligned}
\operatorname{Re}_{L}(z) & =\operatorname{Re}_{2} \operatorname{Li}_{2}\left(z e^{i 0}\right)=-\frac{1}{2} \int_{0}^{z} \frac{\log \left(1-2 x \cos 0+x^{2}\right)}{x} d x \\
& =-\frac{1}{2} \int_{0}^{z} \frac{\log (1-x)^{2}}{x} d x=-\int_{0}^{z} \frac{\log |1-x|}{x} d x=\lambda(z),
\end{aligned}
$$


where $\lambda(z)$ is the same function as in (9). Therefore, we have

$$
\lambda(z)= \begin{cases}\operatorname{Li}_{2}(z), & z \leq 1 \\ -\mathrm{Li}_{2}\left(z^{-1}\right)-\frac{1}{2} \log ^{2} z+\frac{\pi^{2}}{3}, & z>1\end{cases}
$$

By using the following special values [10]

$$
\begin{aligned}
& \mathrm{Li}_{2}\left(a^{2}\right)=\frac{\pi^{2}}{15}-\log ^{2}(-a), \quad \mathrm{Li}_{2}(-a)=\frac{\pi^{2}}{10}-\log ^{2}(-a), \\
& \mathrm{Li}_{2}(a)=-\frac{\pi^{2}}{15}+\frac{1}{2} \log ^{2}(-a), \mathrm{Li}_{2}\left(a^{-1}\right)=-\frac{\pi^{2}}{10}-\log ^{2}(-a) .
\end{aligned}
$$

a straightforward computation gives

$$
\begin{aligned}
\mathscr{L}([i j k \ell]) & =\mathscr{L}(-[i j k m])=\mathscr{L}([i j \ell m])=\mathscr{L}(-[i k \ell m])=\mathscr{L}([j k \ell m]) \\
& =\frac{1}{2}\left(\Lambda\left(a^{2}\right)+\Lambda\left(-a^{-1}\right)+\Lambda\left(a^{-1}\right)\right)=-\frac{\pi^{2}}{20}
\end{aligned}
$$

and

$$
\begin{aligned}
\underline{S}^{i j k \ell m} & =\mathscr{L}([i j k \ell])+\mathscr{L}(-[i j k m])+\mathscr{L}([i j \ell m])+\mathscr{L}(-[i k \ell m])+\mathscr{L}([j k \ell m]) \\
& =-\frac{\pi^{2}}{4}
\end{aligned}
$$

This is, because the expression for $\mathscr{L}([i j k \ell]$ ) (see (9)) changes the sign under the cyclic permutation of indices $(i j k \ell)$ and the solution is invariant under cyclic permutation of indices $(i j k \ell m)$.

Let us now consider the second branch of solutions: one can easily see that

$$
\begin{aligned}
& x_{i j}=x_{j k}=x_{k \ell}=x_{\ell m}=x_{i m}=a^{-1} \\
& x_{i k}=x_{j \ell}=x_{k m}=x_{i \ell}=x_{j m}=-1
\end{aligned}
$$

with

$$
a=\frac{1}{2}-\frac{\sqrt{5}}{2}
$$

is a solution of (14) and (15), because (19) is a solution of (13) and (7). Therefore, on the solution (20) as well as on all other solutions of (14), we have

$$
\underline{S}^{i j k \ell m}=\frac{\pi^{2}}{4}
$$

where we used $\Lambda(z)=\lambda(z)-\lambda\left(z^{-1}\right)$, and, therefore, $\Lambda\left(z^{-1}\right)=-\Lambda(z)$. 
Analogously, we get similar results for the white 4-ambo-simplex $\lceil i j k \ell m\rceil$. Here, the corner equations are:

$$
\frac{\partial \bar{S}^{i j k \ell m}}{\partial x_{i j k}}=\frac{\partial \mathscr{L}\left(T_{k}[i j \ell m]\right)}{\partial x_{i j k}}+\frac{\partial \mathscr{L}\left(-T_{j}[i k \ell m]\right)}{\partial x_{i j k}}+\frac{\partial \mathscr{L}\left(T_{i}[j k \ell m]\right)}{\partial x_{i j k}}=0
$$

and

$$
\frac{\partial \bar{S}^{i j k \ell m}}{\partial x_{i j \ell}}=\frac{\partial \mathscr{L}\left(-T_{\ell}[i j k m]\right)}{\partial x_{i j \ell}}+\frac{\partial \mathscr{L}\left(-T_{j}[i k \ell m]\right)}{\partial x_{i j \ell}}+\frac{\partial \mathscr{L}\left(T_{i}[j k \ell m]\right)}{\partial x_{i j \ell}}=0 .
$$

Explicitly, they read

$$
\frac{1}{x_{i j k}} \log \left|E_{i j k}\right|=0 \text { and } \frac{1}{x_{i j \ell}} \log \left|E_{i j \ell}\right|=0
$$

where

$$
E_{i j k}:=\frac{x_{i j k} x_{k \ell m}+x_{i k m} x_{j k \ell}}{x_{i j k} x_{k \ell m}-x_{i k \ell} x_{j k m}} \cdot \frac{x_{i j k} x_{j \ell m}-x_{i j \ell} x_{j k m}}{x_{i j k} x_{j \ell m}+x_{i j m} x_{j k \ell}} \cdot \frac{x_{i j k} x_{i \ell m}+x_{i j m} x_{i k \ell}}{x_{i j k} x_{i \ell m}-x_{i j \ell} x_{i k m}}
$$

and

$$
E_{i j \ell}:=\frac{x_{i j \ell} x_{k \ell m}-x_{i k \ell} x_{j \ell m}}{x_{i j \ell} x_{k \ell m}+x_{i \ell m} x_{j k \ell}} \cdot \frac{x_{i j \ell} x_{j k m}-x_{i j m} x_{j k \ell}}{x_{i j \ell} x_{j k m}-x_{i j k} x_{j \ell m}} \cdot \frac{x_{i j \ell} x_{i k m}-x_{i j k} x_{i \ell m}}{x_{i j \ell} x_{i k m}-x_{i j m} x_{i k \ell}} .
$$

The analogue of Theorem 3.1 reads:

Theorem 3.3 Every solution of the system (5) solves either the system

$$
\begin{gathered}
E_{i j k}=-1, \quad E_{i j \ell}=-1, \quad E_{i j m}=-1, \quad E_{i k \ell}=-1, \quad E_{i k m}=-1, \\
E_{i \ell m}=-1, \quad E_{j k \ell}=-1, \quad E_{j k m}=-1, \quad E_{j \ell m}=-1, \quad E_{k \ell m}=-1
\end{gathered}
$$

or the system

$$
\begin{gathered}
E_{i j k}=1, \quad E_{i j \ell}=1, \quad E_{i j m}=1, \quad E_{i k \ell}=1, \quad E_{i k m}=1, \\
E_{i \ell m}=1, \quad E_{j k \ell}=1, \quad E_{j k m}=1, \quad E_{j \ell m}=1, \quad E_{k \ell m}=1 .
\end{gathered}
$$

Furthermore the system (22) is equivalent to the system (8) (that is dKP on the corresponding white 4-ambo-simplex). The system (23) is equivalent to the system 


$$
\begin{aligned}
& x_{i k m} x_{i \ell m} x_{j k m} x_{j \ell m}-x_{i j m} x_{i \ell m} x_{j k m} x_{k \ell m}+x_{i j m} x_{i k m} x_{j \ell m} x_{k \ell m}=0, \\
& x_{i j \ell} x_{i j m} x_{i k \ell} x_{i k m}-x_{i j k} x_{i j m} x_{i k \ell} x_{i \ell m}+x_{i j k} x_{i j \ell} x_{i k \ell} x_{i \ell m}=0, \\
& x_{j k m} x_{i j k} x_{j \ell m} x_{i j \ell}-x_{j k \ell} x_{i j k} x_{j \ell m} x_{i j m}+x_{j k \ell} x_{j k m} x_{j \ell m} x_{i j m}=0, \\
& x_{i k \ell} x_{j k \ell} x_{i k m} x_{j k m}-x_{k \ell m} x_{j k \ell} x_{i k m} x_{i j k}+x_{k \ell m} x_{i k \ell} x_{i k m} x_{i j k}=0, \\
& x_{j \ell m} x_{k \ell m} x_{i j \ell} x_{i k \ell}-x_{i \ell m} x_{k \ell m} x_{i j \ell} x_{j k \ell}+x_{i \ell m} x_{j \ell m} x_{i j \ell} x_{j k \ell}=0,
\end{aligned}
$$

which is the system (8) after the transformation $x \mapsto x^{-1}$ of fields (that is $d K P^{-}$on the corresponding white 4-ambo-simplex).

The analogue of Theorem 3.2 reads:

Theorem 3.4 (Closure relation) There holds:

$$
\bar{S}^{i j k \ell m} \pm \frac{\pi^{2}}{4}=0
$$

on all solutions of (22) and (23), respectively. Therefore, one can redefine the 3-form $\mathscr{L}$ as

$$
\tilde{\mathscr{L}}([i j k \ell]):=\mathscr{L}([i j k \ell]) \pm \frac{\pi^{2}}{4}
$$

in order to get $\bar{S}^{i j k \ell m}=0$ on all solutions of (22) and (23), respectively.

\section{The Cubic Lattice $\mathbb{Z}^{N}$}

We will now consider the relation between the elementary cells of the root lattice $Q\left(A_{N}\right)$ and the cubic lattice $\mathbb{Z}^{N}$. The points of $Q\left(A_{N}\right)$ and of $\mathbb{Z}^{N}$ are in a one-to-one correspondence via

$$
P_{i}: Q\left(A_{N}\right) \rightarrow \mathbb{Z}^{N}, \quad x\left(n_{0}, \ldots, n_{i-1}, n_{i}, n_{i+1}, \ldots, n_{N}\right) \mapsto x\left(n_{0}, \ldots, n_{i-1}, n_{i+1}, \ldots, n_{N}\right) .
$$

In the present paper, we will always apply $P_{i}$ with $i<j, k, \ell, \ldots$

We denote by

$$
\{j k \ell\}:=\left\{x, x_{j}, x_{k}, x_{\ell}, x_{j k}, x_{j \ell}, x_{k \ell}, x_{j k \ell}\right\}
$$

the oriented 3D cubes of $\mathbb{Z}^{N}$. We say that the $3 \mathrm{D}$ cube $\{j k \ell\}$ is positively oriented if $j<k<\ell$. Any permutation of two indices changes the orientation to the opposite one. Also in this case, we always write the letters in the brackets in increasing order, so, e.g., in writing $\{j k \ell\}$ we assume that $j<k<\ell$ and avoid the notation $\{k j \ell\}$ or $\{j \ell k\}$ for the negatively oriented $3 \mathrm{D}$ cube $-\{j k \ell\}$. 
(a)

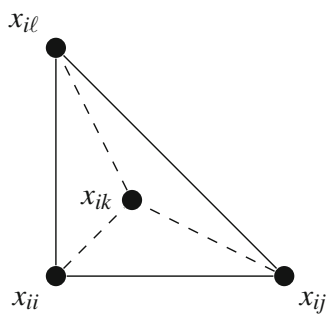

(d)

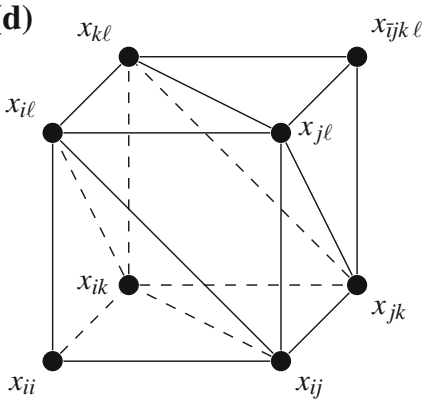

(b)

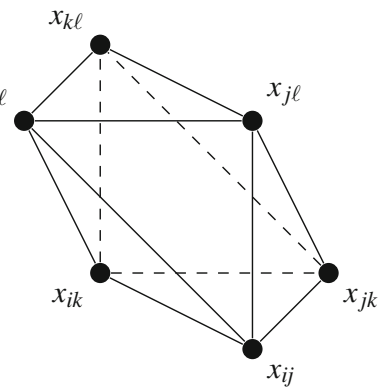

(c)

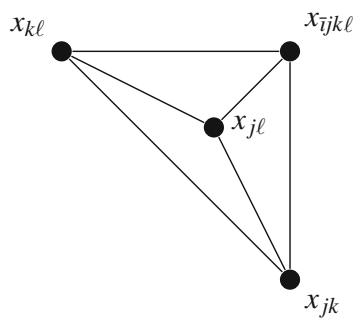

(e)

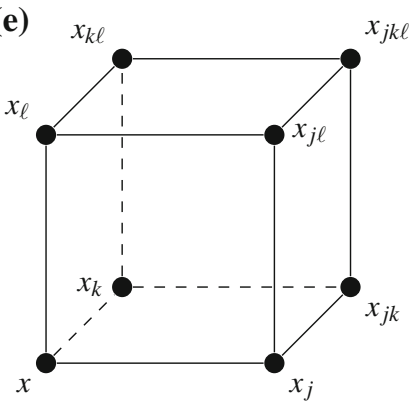

Fig. 2 Three adjacent 3-cells of the lattice $Q\left(A_{N}\right)$ : a black tetrahedron $-T_{i}\lfloor i j k \ell\rfloor$, b octahedron $[i j k \ell], \mathbf{c}$ white tetrahedron $-T_{\bar{l}}\lceil i j k \ell\rceil$. The sum $\mathbf{d}$ of these 3 -cells corresponds to a $3 \mathrm{D}$ cube $\mathbf{e}$

The object in $Q\left(A_{N}\right)$ which corresponds to the $3 \mathrm{D}$ cube $\{j k \ell\}$ is the sum of three adjacent 3 -cells, namely

- the black tetrahedron $-T_{i}\lfloor i j k \ell\rfloor$ (see Fig. 2a),

- the octahedron $[i j k \ell]$ (see Fig. 2b),

- and the white tetrahedron $-T_{\bar{l}}\lceil i j k \ell\rceil$ (see Fig. 2c).

It contains sixteen triangles and to every quadrilateral face of $\{j k l\}$ there corresponds a pair of these triangles containing one black and one white triangle. Here, the map $P_{i}$ reads as follows:

$$
x_{i i} \mapsto x, \quad x_{i j} \mapsto x_{j}, \quad x_{j k} \mapsto x_{j k}, \quad \text { and } \quad x_{\bar{l} j k \ell} \mapsto x_{j k \ell}
$$

As a four-dimensional elementary cell of $\mathbb{Z}^{N}$, we consider an oriented $4 \mathrm{D}$ cube

$$
\{j k \ell m\}:=\left\{x, x_{j}, x_{k}, x_{\ell}, x_{m}, x_{j k}, x_{j \ell}, x_{j m}, x_{k \ell}, x_{k m}, x_{\ell m}, x_{j k \ell}, x_{j k m}, x_{j \ell m}, x_{k \ell m}, x_{j k \ell m}\right\}
$$

The 4D cube $\{j k \ell m\}$ corresponds to the sum of four 4-cells in $Q\left(A_{N}\right)$ :

- the black 4-simplex $-T_{i} \llbracket i j k \ell m \rrbracket$,

- the black 4-ambo-simplex $\lfloor j k \ell \ell m\rfloor$, 


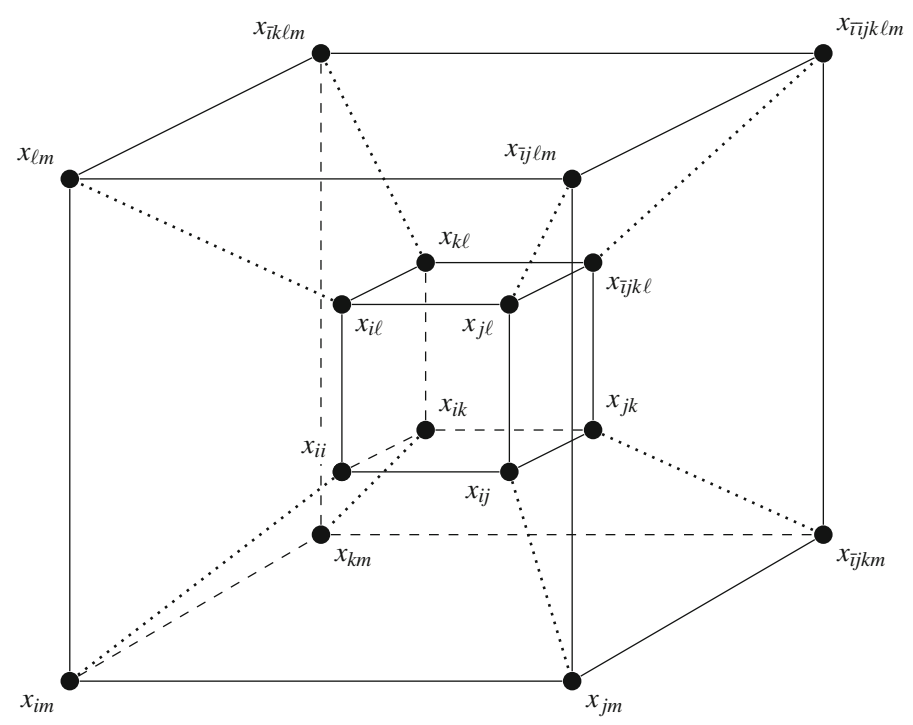

Fig. 3 The sum of the black 4-simplex $-T_{i}\lfloor i j k \ell m \rrbracket$, the adjacent black 4-ambo-simplex $\lfloor i j k \ell m\rfloor$, the adjacent white 4-ambo-simplex $-T_{\bar{l}}\lceil i j k \ell m\rceil$, and the adjacent white 4-simplex $\left.T_{\bar{l}} T_{\bar{l}} \llbracket i j k \ell m\right\rceil$ corresponds to the $4 \mathrm{D}$ cube $\{j k \ell m\}$

- the white 4-ambo-simplex $-T_{\bar{l}}\lceil i j k \ell m\rceil$, and

- the white 4-simplex $T_{i} T_{i} \llbracket i j k \ell m \rrbracket$

(see Fig. 3). It contains sixteen tetrahedra (eight black and eight white ones) and eight octahedra. Here, the map $P_{i}$ reads as follows:

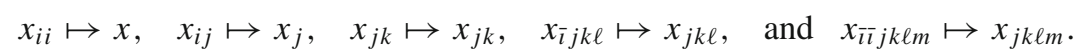

Also in the cubic case there is an easy recipe to obtain the orientation of the facets of an (oriented) 4D cube: on every index between the brackets we put alternately a "+" and a "-" starting with a "+" on the last index. Then we get each facet by deleting one index and putting the corresponding sign in front of the bracket. For instance., the $4 \mathrm{D}$ cube

$$
\left\{\begin{array}{c}
-+ \\
j \quad k \quad l \\
k
\end{array}\right\}
$$

has the eight 3D facets: $\{j k \ell\},-\{j k m\},\{j \ell m\},-\{k \ell m\}$ and the opposite ones $-T_{m}\{j k \ell\}, T_{\ell}\{j k m\},-T_{k}\{j \ell m\}$, and $T_{j}\{k \ell m\}$.

As a consequence of Definition 2.2, in each flower in $\mathbb{Z}^{N}$, every 3D cube has exactly four adjacent $3 \mathrm{D}$ cubes.

We will now prove the analogue of Theorem 2.5. This proof is easier than the one for $Q\left(A_{N}\right)$, because of the simpler combinatorial structure. 
Theorem 4.1 The flower at any interior vertex of any 3-manifold in $\mathbb{Z}^{N}$ can be represented as a sum of $4 D$ corners in $\mathbb{Z}^{N+1}$.

Proof Set $M:=N+1$ and consider the flower of an interior vertex $x$ of an arbitrary 3-manifold in $\mathbb{Z}^{N}$. Over each $3 \mathrm{D}$ corner $\{j k \ell\}$ (petal) of the flower, we can build a $4 \mathrm{D}$ corner adjacent to $x$ on the 4D cube $\{j k \ell M\}$. Then the vertical 3D cubes coming from two successive petals of the flower carry opposite orientations, so that all vertical squares cancel away from the sum of the 4D corners.

Let $\mathfrak{L}$ be a discrete 3 -form on $\mathbb{Z}^{N}$. The exterior derivative $d \mathfrak{L}$ is a discrete 4-form whose value at any $4 \mathrm{D}$ cube in $\mathbb{Z}^{N}$ is the action functional of $\mathfrak{L}$ on the 3 -manifold consisting of the facets of the $4 \mathrm{D}$ cube:

$$
\begin{array}{r}
S^{j k \ell m}:=d \mathfrak{L}(\{j k \ell m\})=\mathfrak{L}(\{j k \ell\})+\mathfrak{L}(-\{j k m\})+\mathfrak{L}(\{j \ell m\})+\mathfrak{L}(-\{k \ell m\}) \\
+\mathfrak{L}\left(-T_{m}\{j k \ell\}\right)+\mathfrak{L}\left(T_{\ell}\{j k m\}\right)+\mathfrak{L}\left(-T_{k}\{j \ell m\}\right)+\mathfrak{L}\left(T_{j}\{k \ell m\}\right) .
\end{array}
$$

Accordingly, the Euler-Lagrange equations on the 4D cube $\{j k \ell m\}$ are given by

$$
\begin{aligned}
& \frac{\partial S^{j k \ell m}}{\partial x}=0 \\
& \frac{\partial S^{j k \ell m}}{\partial x_{j}}=0, \frac{\partial S^{j k \ell m}}{\partial x_{k}}=0, \frac{\partial S^{j k \ell m}}{\partial x_{\ell}}=0, \frac{\partial S^{j k \ell m}}{\partial x_{m}}=0 \\
& \frac{\partial S^{j k \ell m}}{\partial x_{j k}}=0, \frac{\partial S^{j k \ell m}}{\partial x_{j \ell}}=0, \frac{\partial S^{j k \ell m}}{\partial x_{j m}}=0, \frac{\partial S^{j k \ell m}}{\partial x_{k \ell}}=0, \frac{\partial S^{j k \ell m}}{\partial x_{k m}}=0, \frac{\partial S^{j k \ell m}}{\partial x_{\ell m}}=0 \\
& \frac{\partial S^{j k \ell m}}{\partial x_{j k \ell}}=0, \frac{\partial S^{j k \ell m}}{\partial x_{j k m}}=0, \frac{\partial S^{j k \ell m}}{\partial x_{j \ell m}}=0, \frac{\partial S^{j k \ell m}}{\partial x_{k \ell m}}=0 \\
& \frac{\partial S^{j k \ell m}}{\partial x_{j k \ell m}}=0 .
\end{aligned}
$$

They are called corner equations.

The following statement is an immediate consequence of Theorem 4.1:

Theorem 4.2 For every discrete 3-form on $\mathbb{Z}^{N}$ and every 3-manifold in $\mathbb{Z}^{N}$ all corresponding Euler-Lagrange equations can be written as a sum of corner equations.

\section{The dKP Equation on $\mathbb{Z}^{N}$}

On the $3 \mathrm{D}$ cube $\{j k \ell\}$ in $\mathbb{Z}^{3}(j<k<\ell)$ we put the equation

$$
x_{j} x_{k \ell}-x_{k} x_{j \ell}+x_{\ell} x_{j k}=0 .
$$

We can extend this system in a consistent way (see [1]) to the four-dimensional cubic lattice $\mathbb{Z}^{4}$ and its higher-dimensional analogues, such that the eight facets $\{j k \ell\}$, 
$-\{j k m\},\{j \ell m\},-\{k \ell m\},-T_{m}\{j k \ell\}, T_{\ell}\{j k m\},-T_{k}\{j \ell m\}, T_{j}\{k \ell m\}$ of a $4 \mathrm{D}$ cube $\{j k \ell m\}$ carry the equations

$$
\begin{array}{ll}
x_{j} x_{k \ell}-x_{k} x_{j \ell}+x_{\ell} x_{j k}=0, & x_{j m} x_{k \ell m}-x_{k m} x_{j \ell m}+x_{\ell m} x_{j k m}=0, \\
x_{j} x_{k m}-x_{k} x_{j m}+x_{m} x_{j k}=0, & x_{j k} x_{k \ell m}-x_{k \ell} x_{j k m}+x_{k m} x_{j k \ell}=0, \\
x_{j} x_{\ell m}-x_{\ell} x_{j m}+x_{m} x_{j \ell}=0, & x_{j \ell} x_{k \ell m}-x_{k \ell} x_{j \ell m}+x_{\ell m} x_{j k \ell}=0, \\
x_{k} x_{\ell m}-x_{\ell} x_{k m}+x_{m} x_{k \ell}=0, & x_{j k} x_{j \ell m}-x_{j \ell} x_{j k m}+x_{j m} x_{j k \ell}=0 .
\end{array}
$$

Note that, in the four equations in the left column, the fields with one index always appear with increasing order of indices. The equations in the right column are shifted copies of the ones in the left column. One can derive the system (27) from the system of dKP equations (7) on the black 4-ambo-simplex $\lfloor i j k \ell m\rfloor$ and the system of dKP equations (8) on the white 4-ambo-simplex $T_{\bar{l}}\lceil i j k m \ell\rceil$, by removing the equations on the octahedra $[j k \ell m]$ and $[j k m \ell]$, respectively, from both systems and applying the transformation $P_{i}$ to the fields in the remaining eight equations.

We propose the discrete 3 -form $\mathfrak{L}$ defined as

$$
\mathfrak{L}:=\left(P_{i}\right)_{\star} \mathscr{L}
$$

where $\mathscr{L}$ is the discrete 3 -form on the root lattice $Q\left(A_{N}\right)$ (see (9)). Therefore, $\mathfrak{L}$ evaluated at the $3 \mathrm{D}$ cube $\{j k \ell\}$ reads as

$$
\begin{aligned}
\mathfrak{L}(\{j k \ell\}) & =\left(\left(P_{i}\right)_{\star} \mathscr{L}\right)\left(P_{i}\left(-T_{i}\lfloor i j k \ell\rfloor+[i j k \ell]-T_{\bar{l}}\lceil i j k \ell\rceil\right)\right) \\
& =\left(P_{i}\right)_{\star}(\underbrace{\mathscr{L}\left(-T_{i}\lfloor i j k \ell\rfloor\right)}_{=0}+\mathscr{L}([i j k \ell])-\underbrace{\mathscr{L}\left(-T_{\bar{l}}\lceil i j k \ell\rceil\right)}_{=0})=\left(P_{i}\right)_{\star} \mathscr{L}([i j k \ell]) .
\end{aligned}
$$

For this discrete 3 -form, there are no corner equations on the 4D cube $\{j k \ell m\}$ centered at $x$ and $x_{j k \ell m}$ since $S^{j k \ell m}$ does not depend on these two variables. The remaining corner equations from (25) are given by

$$
\begin{aligned}
\frac{\partial S^{j k \ell m}}{\partial x_{j}} & =\frac{\partial \mathfrak{L}(\{j k \ell\})}{\partial x_{j}}+\frac{\partial \mathfrak{L}(-\{j k m\})}{\partial x_{j}}+\frac{\partial \mathfrak{L}(\{j \ell m\})}{\partial x_{j}}+\underbrace{\frac{\partial \mathfrak{L}\left(T_{j}\{k \ell m\}\right)}{\partial x_{j}}}_{\equiv 0} \\
& =\left(P_{i}\right)_{\star}\left(\frac{\partial \mathscr{L}([i j k \ell])}{\partial x_{i j}}+\frac{\partial \mathscr{L}(-[i j k m])}{\partial x_{i j}}+\frac{\partial \mathscr{L}([i j \ell m])}{\partial x_{i j}}\right)=\frac{1}{x_{j}} \log \left|\mathscr{E}_{j}\right|=0, \\
\frac{\partial S^{j k \ell m}}{\partial x_{j k}} & =\frac{\partial \mathfrak{L}(\{j k \ell\})}{\partial x_{j k}}+\frac{\partial \mathfrak{L}(-\{j k m\})}{\partial x_{j k}}+\frac{\partial \mathfrak{L}\left(-T_{k}\{j \ell m\}\right)}{\partial x_{j k}}+\frac{\partial \mathfrak{L}\left(T_{j}\{k \ell m\}\right)}{\partial x_{j k}} \\
& =\left(P_{i}\right)_{\star}\left(\frac{\partial \mathscr{L}([i j k \ell])}{\partial x_{j k}}+\frac{\partial \mathscr{L}(-[i j k m])}{\partial x_{j k}}+\frac{\partial \mathscr{L}\left(-T_{\bar{l}} T_{k}[i j \ell m]\right)}{\partial x_{j k}}\right. \\
& \left.+\frac{\partial \mathscr{L}\left(T_{\bar{l}} T_{j}[i k \ell m]\right)}{\partial x_{j k}}\right) \\
= & \frac{1}{x_{j k}} \log \left|\frac{\mathscr{E}_{j k}}{\overline{\mathscr{E}}_{j k}}\right|=0,
\end{aligned}
$$




$$
\begin{aligned}
\frac{\partial S^{j k \ell m}}{\partial x_{j k \ell}} & =\underbrace{\frac{\partial \mathfrak{L}(\{j k \ell\})}{\partial x_{j k \ell}}}_{\equiv 0}+\frac{\partial \mathfrak{L}\left(T_{\ell}\{j k m\}\right)}{\partial x_{j k \ell}}+\frac{\partial \mathfrak{L}\left(-T_{k}\{j \ell m\}\right)}{\partial x_{j k \ell}}+\frac{\partial \mathfrak{L}\left(T_{j}\{k \ell m\}\right)}{\partial x_{j k \ell}} \\
& =\left(P_{i}\right)_{\star}\left(\frac{\partial \mathscr{L}\left(T_{\bar{l}} T_{\ell}[i j k m]\right)}{\partial x_{\bar{l}} j k \ell}+\frac{\partial \mathscr{L}\left(-T_{\bar{l}} T_{k}[i j \ell m]\right)}{\partial x_{\bar{l}} j k \ell}+\frac{\partial \mathscr{L}\left(T_{\bar{l}} T_{j}[i k \ell m]\right)}{\partial x_{\bar{l}} j k \ell}\right) \\
& =\frac{1}{x_{j k \ell}} \log \left|\frac{1}{\mathscr{E}_{j k \ell}}\right|=0,
\end{aligned}
$$

where

$$
\mathscr{E}_{j}:=\left(P_{i}\right)_{\star} E_{i j}, \quad \underline{\mathscr{E}}_{j k}:=\left(P_{i}\right)_{\star} E_{j k}, \quad \overline{\mathscr{E}}_{j k}:=\left(P_{i}\right)_{\star} E_{i j k}, \quad \text { and } \quad \mathscr{E}_{j k \ell}:=\left(P_{i}\right)_{\star} E_{j k \ell}
$$

Hereafter, we only consider solutions, where all fields are non-zero (we call these solutions non-singular). As in the case of the root lattice $Q\left(A_{N}\right)$ every corner equation has two classes of solutions.

Theorem 5.1 Every solution of the system (25) solves either the system

$$
\begin{aligned}
& \mathscr{E}_{j}=-1, \quad \mathscr{E}_{k}=-1, \quad \mathscr{E}_{\ell}=-1, \quad \mathscr{E}_{m}=-1, \\
& \underline{\mathscr{E}}_{j k}=-1, \quad \mathscr{\mathscr { E }}_{j \ell}=-1, \quad \underline{\mathscr{E}}_{j m}=-1, \quad \underline{\mathscr{E}}_{k \ell}=-1, \quad \underline{\mathscr{E}}_{k m}=-1, \quad \mathscr{\mathscr { E }}_{\ell m}=-1, \\
& \overline{\mathscr{E}}_{j k}=-1, \quad \overline{\mathscr{E}}_{j \ell}=-1, \quad \overline{\mathscr{E}}_{j m}=-1, \quad \overline{\mathscr{E}}_{k \ell}=-1, \quad \overline{\mathscr{E}}_{k m}=-1, \quad \overline{\mathscr{E}}_{\ell m}=-1, \\
& \mathscr{E}_{j k \ell}=-1, \quad \mathscr{E}_{j k m}=-1, \quad \mathscr{E}_{j \ell m}=-1, \quad \mathscr{E}_{k \ell m}=-1
\end{aligned}
$$

or the system

$$
\begin{aligned}
& \mathscr{E}_{j}=1, \quad \mathscr{E}_{k}=1, \quad \mathscr{E}_{\ell}=1, \quad \mathscr{E}_{m}=1, \\
& \mathscr{\mathscr { E }}_{j k}=1, \quad \underline{\mathscr{E}}_{j \ell}=1, \quad \underline{\mathscr{E}}_{j m}=1, \quad \underline{\mathscr{E}}_{k \ell}=1, \quad \underline{\mathscr{E}}_{k m}=1, \quad \underline{\mathscr{E}}_{\ell m}=1, \overline{\mathscr{E}}_{j k}=1, \quad \overline{\mathscr{E}}_{j \ell}=1, \quad \overline{\mathscr{E}}_{j m}=1, \quad \overline{\mathscr{E}}_{k \ell}=1, \quad \overline{\mathscr{E}}_{k m}=1, \quad \overline{\mathscr{E}}_{\ell m}=1, \\
& \overline{\mathscr{E}}_{j k \ell}=1, \quad \mathscr{\mathscr { E }}_{j k m}=1, \quad \mathscr{E}_{j \ell m}=1, \quad \mathscr{E}_{k \ell m}=1 .
\end{aligned}
$$

Furthermore the system (29) is equivalent to the system (27) (this is dKP on the corresponding $4 \mathrm{D}$ cube). The system (30) is equivalent to the system

$$
\begin{aligned}
& x_{k} x_{\ell} x_{j k} x_{j \ell}-x_{j} x_{\ell} x_{j k} x_{k \ell}+x_{j} x_{k} x_{j \ell} x_{k \ell}=0, \\
& x_{k} x_{m} x_{j k} x_{j m}-x_{j} x_{m} x_{j k} x_{k m}+x_{j} x_{k} x_{j m} x_{k m}=0, \\
& x_{\ell} x_{m} x_{j \ell} x_{j m}-x_{j} x_{m} x_{j \ell} x_{\ell m}+x_{j} x_{\ell} x_{j m} x_{\ell m}=0, \\
& x_{\ell} x_{m} x_{k \ell} x_{k m}-x_{k} x_{m} x_{k \ell} x_{\ell m}+x_{k} x_{\ell} x_{k m} x_{\ell m}=0, \\
& x_{k m} x_{\ell m} x_{j k m} x_{j \ell m}-x_{j m} x_{\ell m} x_{j k m} x_{k \ell m}+x_{j m} x_{k m} x_{j \ell m} x_{k \ell m}=0, \\
& x_{k \ell} x_{\ell m} x_{j k \ell} x_{j \ell m}-x_{j \ell} x_{\ell m} x_{j k \ell} x_{k \ell m}+x_{j \ell} x_{k \ell} x_{j \ell m} x_{k \ell m}=0, \\
& x_{k \ell} x_{k m} x_{j k \ell} x_{j k m}-x_{j k} x_{k m} x_{j k \ell} x_{k \ell m}+x_{j k} x_{k \ell} x_{j k m} x_{k \ell m}=0, \\
& x_{j \ell} x_{j m} x_{j k \ell} x_{j k m}-x_{j k} x_{j m} x_{j k \ell} x_{j \ell m}+x_{j k} x_{j \ell} x_{j k m} x_{j \ell m}=0,
\end{aligned}
$$


which is the system (27) after the transformation $x \mapsto x^{-1}$ of fields (this is dKP $P^{-}$on the corresponding $4 \mathrm{D}$ cube).

Proof Let $x$ be a solution of the system (25) such that $\mathscr{E}_{j}=-1$ and $\mathscr{E}_{k}=-1$. Then we know from the proof of Theorem 3.1 that

$$
\begin{aligned}
\mathscr{E}_{j}=-1, \quad \mathscr{E}_{k}=-1, \quad \mathscr{E}_{\ell}=-1, \quad \mathscr{E}_{m}=-1, \\
\underline{\mathscr{E}}_{j k}=-1, \quad \underline{\mathscr{E}}_{j \ell}=-1, \quad \underline{\mathscr{E}}_{j m}=-1, \quad \underline{\mathscr{E}}_{k \ell}=-1, \quad \underline{\mathscr{E}}_{k m}=-1, \quad \underline{\mathscr{E}}_{l m}=-1
\end{aligned}
$$

and that the latter system is equivalent to

$$
\begin{aligned}
& x_{j} x_{k \ell}-x_{k} x_{j \ell}+x_{\ell} x_{j k}=0, \\
& x_{j} x_{k m}-x_{k} x_{j m}+x_{m} x_{j k}=0, \\
& x_{j} x_{\ell m}-x_{\ell} x_{j m}+x_{m} x_{j \ell}=0, \\
& x_{k} x_{\ell m}-x_{\ell} x_{k m}+x_{m} x_{k \ell}=0, \\
& x_{j k} x_{\ell m}-x_{j \ell} x_{k m}+x_{j m} x_{k \ell}=0 .
\end{aligned}
$$

On the other hand, if we consider a solution $x$ of (25) such that $\mathscr{E}_{j}=1$ and $\mathscr{E}_{k}=1$, we know from the proof of Theorem 3.1 that

$$
\begin{aligned}
\mathscr{E}_{j} & =1, \quad \mathscr{E}_{k}=1, \quad \mathscr{E}_{\ell}=1, \quad \mathscr{E}_{m}=1, \\
\underline{\mathscr{E}}_{j k} & =1, \quad \underline{\mathscr{E}}_{j \ell}=1, \quad \underline{\mathscr{E}}_{j m}=1, \quad \underline{\mathscr{E}}_{k \ell}=1, \quad \underline{\mathscr{E}}_{k m}=1, \quad \underline{\mathscr{E}}_{l m}=1
\end{aligned}
$$

and that the latter system is equivalent to

$$
\begin{aligned}
& x_{k} x_{\ell} x_{j k} x_{j \ell}-x_{j} x_{\ell} x_{j k} x_{k \ell}+x_{j} x_{k} x_{j \ell} x_{k \ell}=0, \\
& x_{k} x_{m} x_{j k} x_{j m}-x_{j} x_{m} x_{j k} x_{k m}+x_{j} x_{k} x_{j m} x_{k m}=0, \\
& x_{\ell} x_{m} x_{j \ell} x_{j m}-x_{j} x_{m} x_{j \ell} x_{\ell m}+x_{j} x_{\ell} x_{j m} x_{\ell m}=0, \\
& x_{\ell} x_{m} x_{k \ell} x_{k m}-x_{k} x_{m} x_{k \ell} x_{\ell m}+x_{k} x_{\ell} x_{k m} x_{\ell m}=0, \\
& x_{j \ell} x_{j m} x_{k \ell} x_{k m}-x_{j k} x_{j m} x_{k \ell} x_{\ell m}+x_{j k} x_{j \ell} x_{k m} x_{\ell m}=0 .
\end{aligned}
$$

Now, let $x$ be a solution of the system (25) such that $\mathscr{E}_{j k \ell}=-1$ and $\mathscr{E}_{j k m}=-1$. Then we know from the proof of Theorem 3.3 that

$$
\begin{aligned}
& \overline{\mathscr{E}}_{j k}=1, \quad \overline{\mathscr{E}}_{j \ell}=1, \quad \overline{\mathscr{E}}_{j m}=1, \quad \overline{\mathscr{E}}_{k \ell}=1, \quad \overline{\mathscr{E}}_{k m}=1, \quad \overline{\mathscr{E}}_{\ell m}=1, \\
& \mathscr{E}_{j k \ell}=1, \quad \mathscr{E}_{j k m}=1, \quad \mathscr{E}_{j \ell m}=1, \quad \mathscr{E}_{k \ell m}=1
\end{aligned}
$$

and that the latter system is equivalent to 


$$
\begin{aligned}
& x_{\ell m} x_{j k m}-x_{k m} x_{j \ell m}+x_{j m} x_{k \ell m}=0, \\
& x_{k m} x_{j k \ell}-x_{k \ell} x_{j k m}+x_{j k} x_{k \ell m}=0, \\
& x_{\ell m} x_{j k \ell}-x_{k \ell} x_{j \ell m}+x_{j \ell} x_{k \ell m}=0, \\
& x_{j m} x_{j k \ell}-x_{j \ell} x_{j k m}+x_{j k} x_{j \ell m}=0, \\
& x_{j k} x_{\ell m}-x_{j \ell} x_{k m}+x_{j m} x_{k \ell}=0 .
\end{aligned}
$$

On the other hand, if we consider a solution $x$ of (25) such that $\mathscr{E}_{j}=1$ and $\mathscr{E}_{k}=1$, we know from the proof of Theorem 3.3 that

$$
\begin{aligned}
& \overline{\mathscr{E}}_{j k}=1, \quad \overline{\mathscr{E}}_{j \ell}=1, \quad \overline{\mathscr{E}}_{j m}=1, \quad \overline{\mathscr{E}}_{k \ell}=1, \quad \overline{\mathscr{E}}_{k m}=1, \quad \overline{\mathscr{E}}_{\ell m}=1, \\
& \mathscr{E}_{j k \ell}=1, \quad \mathscr{E}_{j k m}=1, \quad \mathscr{E}_{j \ell m}=1, \quad \mathscr{E}_{k \ell m}=1
\end{aligned}
$$

and that the latter system is equivalent to

$$
\begin{aligned}
& x_{k m} x_{\ell m} x_{j k m} x_{j \ell m}-x_{j m} x_{\ell m} x_{j k m} x_{k \ell m}+x_{j m} x_{k m} x_{j \ell m} x_{k \ell m}=0, \\
& x_{k \ell} x_{\ell m} x_{j k \ell} x_{j \ell m}-x_{j \ell} x_{\ell m} x_{j k \ell} x_{k \ell m}+x_{j \ell} x_{k \ell} x_{j \ell m} x_{k \ell m}=0, \\
& x_{k \ell} x_{k m} x_{j k \ell} x_{j k m}-x_{j k} x_{k m} x_{j k \ell} x_{k \ell m}+x_{j k} x_{k \ell} x_{j k m} x_{k \ell m}=0, \\
& x_{j \ell} x_{j m} x_{j k \ell} x_{j k m}-x_{j k} x_{j m} x_{j k \ell} x_{j \ell m}+x_{j k} x_{j \ell} x_{j k m} x_{j \ell m}=0, \\
& x_{j \ell} x_{j m} x_{k \ell} x_{k m}-x_{j k} x_{j m} x_{k \ell} x_{\ell m}+x_{j k} x_{j \ell} x_{k m} x_{\ell m}=0 .
\end{aligned}
$$

Since a solution $x$ of (25) cannot solve

$$
x_{j k} x_{\ell m}-x_{j \ell} x_{k m}+x_{j m} x_{k \ell}=0
$$

and

$$
x_{j \ell} x_{j m} x_{k \ell} x_{k m}-x_{j k} x_{j m} x_{k \ell} x_{\ell m}+x_{j k} x_{j \ell} x_{k m} x_{\ell m}=0
$$

at the same time, this proves the theorem.

Theorem 5.2 (Closure relation) There holds $S^{j k \ell m}=0$ on all solutions of (25).

Proof Let $x$ be a solution of (29) or (30). Then

$$
\begin{aligned}
S^{j k \ell m} & =d \mathfrak{L}(\{j k \ell\})=\left(P_{i}\right)_{\star}(d \mathscr{L}(\lfloor i j k \ell m\rfloor)+d \mathscr{L}(-\lceil i j k \ell m\rceil))=\underline{S}^{i j k \ell m}-\bar{S}^{i j k \ell m} \\
& = \pm \frac{\pi^{2}}{4} \mp \frac{\pi^{2}}{4}=0
\end{aligned}
$$

due to Theorems 3.2 and 3.4 since every solution of (29) solves (13) and (22) after the transformation $P_{i}$ of variables and every solution of (30) solves (14) and (23) after the transformation $P_{i}$ of variables. 


\section{Conclusion}

The fact that the three-dimensional (hyperbolic) dKP equation is, in a sense, equivalent to the Euler-Lagrange equations of the corresponding action is rather surprising since for the two-dimensional (hyperbolic) quad-equations an analogous statement is not true (see [4, 6] for more details). On the other hand, in the continuous situation there is an example of a 2-form whose Euler-Lagrange equations are equivalent to the set of equations consisting of the (hyperbolic) sine-Gordon equation and the (evolutionary) modified Korteweg-de Vries equation (see [16] for more details). So, the general picture remains unclear.

In particular, the variational formulation for the other equations of octahedron type in the classification of [1] is still an open problem.

Acknowledgments This research was supported by the DFG Collaborative Research Center TRR 109 "Discretization in Geometry and Dynamics".

\section{Appendix 1: Facets of $N$-Cells of the Root Lattice $Q\left(A_{N}\right)$}

Facets of 3-cells:

Black tetrahedra $\lfloor i j k \ell\rfloor$ : four black triangles $\lfloor i j k\rfloor,-\lfloor i j \ell\rfloor,\lfloor i k \ell\rfloor$, and $-\lfloor j k \ell\rfloor$;

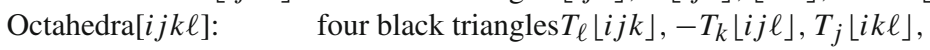
and $-T_{i}\lfloor j k \ell\rfloor$,

four white triangles $\lceil i j k\rceil,-\lceil i j \ell\rceil$, $i k \ell\rceil$, and $-\lceil j k \ell\rceil$;

White tetrahedra $\lceil i j k \ell\rceil$ : four white triangles $T_{\ell}\lceil i j k\rceil,-T_{k}\lceil i j \ell\rceil, T_{j}\lceil i k \ell\rceil$,

Facets of 4-cells: and $-T_{i}\lceil j k \ell\rceil$;

Black 4-simplices $\llbracket i j k \ell m \rrbracket: \quad$ fiveblacktetrahedra $\lfloor i j k \ell\rfloor,-\lfloor i j k m\rfloor,\lfloor i j \ell m\rfloor$, $-\lfloor i k \ell m\rfloor$, and $\lfloor j k \ell m\rfloor$;

Black 4-ambo-simplices $\lfloor i j k \ell m\rfloor$ : five black tetrahedra $T_{m}\lfloor i j k \ell\rfloor,-T_{\ell}\lfloor i j k m\rfloor$, $T_{k}\lfloor i j \ell m\rfloor,-T_{j}\lfloor i k \ell m\rfloor$, and $T_{i}\lfloor j k \ell m\rfloor$,

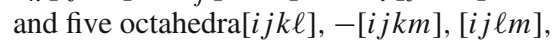
$-[i k \ell m]$, and $[j k \ell m]$;

White 4-ambo-simplices $\lceil i j k \ell m\rceil$ : five octahedra $T_{m}[i j k \ell],-T_{\ell}[i j k m], T_{k}[i j \ell m]$, $-T_{j}[i k \ell m]$, and $T_{i}[j k \ell m]$, and five white tetrahedra $\lceil i j k \ell\rceil,-\lceil i j k m\rceil$, $\lceil i j \ell m\rceil,-\lceil i k \ell m\rceil$, and $\lceil j k \ell m\rceil$;

White 4-simplices $\llbracket i j k \ell m \rrbracket: \quad$ five white tetrahedra $T_{m}\lceil i j k \ell\rceil,-T_{\ell}\lceil i j k m\rceil$, $T_{k}\lceil i j \ell m\rceil,-T_{j}\lceil i k \ell m\rceil$, and $T_{i}\lceil j k \ell m\rceil$. 


\section{Appendix 2: 4D Corners on 4-Cells of the Root Lattice $Q\left(A_{N}\right)$}

Black 4-simplex $\llbracket i j k \ell m \rrbracket$ :

The 4D corner with center vertex $x_{i}$ contains

- the four black tetrahedra $\lfloor i j k \ell\rfloor,-\lfloor i j k m\rfloor,\lfloor i j \ell m\rfloor$, and $-\lfloor i k \ell m\rfloor$;

Black 4-ambo-simplex $\lfloor i j k \ell m\rfloor$ :

The 4D corner with center vertex $x_{i j}$ contains

- the two black tetrahedra $-T_{j}\lfloor i k \ell m\rfloor$, and $T_{i}\lfloor j k \ell m\rfloor$,

- and the three octahedra $[i j k \ell],-[i j k m]$, and $[i j \ell m]$;

White 4-ambo-simplex $\lceil i j k \ell m\rceil$ :

The 4D corner with center vertex $x_{i j k}$ contains

- the three octahedra $T_{k}[i j \ell m],-T_{j}[i k \ell m]$, and $T_{i}[j k \ell m]$,

- and the two white tetrahedra $\lceil i j k \ell\rceil$, and - $\lceil i j k m\rceil$;

White 4-simplex $\llbracket i j k \ell m \rrbracket$ :

The 4D corner with center vertex $x_{i j k \ell}$ contains

- the four white tetrahedra $-T_{\ell}\lceil i j k m\rceil, T_{k}\lceil i j \ell m\rceil,-T_{j}\lceil i k \ell m\rceil$, and $T_{i}\lceil j k \ell m\rceil$.

\section{Appendix 3: Proof of Theorem 2.4}

Set $M:=N+1$ and $L:=N+2$. Then, for the construction of the sum $\Sigma$ of 4 D corners representing the flower $\sigma$ centered in $X$, we use the following algorithm:

(i) For every black tetrahedron $\pm\lfloor i j k \ell\rfloor \in \sigma$ at the interior vertex $X$ we add the $4 \mathrm{D}$ corner with center vertex $X$ on the black 4-simplex $\pm \llbracket i j k \ell M \rrbracket$ to $\Sigma$.

(ii) For every octahedron $\pm[i j k \ell] \in \sigma$ we add the $4 \mathrm{D}$ corner with center vertex $X$ on the black 4-ambo-simplex $\pm\lfloor i j k \ell M\rfloor$ to $\Sigma$.

(iii) For every white tetrahedron $\pm\lceil i j k \ell\rceil \in \sigma$ we add the 4D corner with center vertex $X$ on the white 4-ambo-simplex $\pm\lceil i j k \ell M\rceil$ to $\Sigma$.

(iv) For every white tetrahedron $\pm\lceil i j k M\rceil \in \Sigma \backslash \sigma$ which appeared in $\Sigma$ during the previous step we add the 4D corner with center vertex $X$ on the white 4-simplex $\mp T_{\bar{L}} \llbracket i j k M L \rrbracket$ to $\Sigma$.

Therefore, we have to prove that $\Sigma=\sigma$.

Assume that $X=x_{i}$. Then for each black tetrahedron $\pm\lfloor i j k \ell\rfloor \in \sigma$ we added the three black tetrahedra $\mp\lfloor i j k M\rfloor, \pm\lfloor i j \ell M\rfloor$, and $\mp\lfloor i k \ell M\rfloor$ to $\Sigma$ which do not belong to $\sigma$. Moreover, $\pm\lfloor i j k \ell\rfloor$ has three black triangular facets adjacent to $x_{i}$, namely $\pm\lfloor i j k\rfloor$, which is the common triangle with $\mp\lfloor i j k M\rfloor$ (up to orientation), $\mp\lfloor i j \ell\rfloor$, which is the common triangle with $\pm\lfloor i j \ell M\rfloor$, and $\pm\lfloor i k \ell\rfloor$, which is the common triangle with $\mp\lfloor i k \ell M\rfloor$. Therefore, each of these black tetrahedra has to 
cancel away with the corresponding black tetrahedra from the 4D corner which is coming from the 3 -cell adjacent to $\pm\lfloor i j k \ell\rfloor$ via the corresponding black triangle.

Assume that $X=x_{i j}$. Then for each octahedron $\pm[i j k \ell] \in \sigma$ we added the two black tetrahedra $\mp T_{j}\lfloor i k \ell M\rfloor$ and $\pm T_{i}\lfloor j k \ell M\rfloor$ as well as the two octahedra $\mp[i j k M]$ and $\pm[i j \ell M]$ to $\Sigma$ which do not belong to $\sigma$. Moreover, $\pm[i j k \ell]$ has two black tetrahedral facets adjacent to $x_{i j}$, namely $\pm\lfloor i j k\rfloor$, which is the common triangle with $\mp T_{j}\lfloor i k \ell M\rfloor$, and $\mp\lfloor i j \ell\rfloor$, which is the common triangle with $\pm T_{i}\lfloor j k \ell M$, as well as two white tetrahedral facets adjacent to $x_{i j}$, namely $\pm T_{j}\lceil i k \ell\rceil$, which is the common triangle with $\mp[i j k M]$ and $\pm[i j \ell M]$, and $\mp\lceil j k \ell\rceil$, which is the common triangle with $\pm[i j \ell M]$. Therefore, each of the black tetrahedra $\mp T_{j}\lfloor i k \ell M\rfloor$ and $\pm T_{i}\lfloor j k \ell M$ has to cancel away with the corresponding black tetrahedron from the 4D corner which is coming from the 3 -cell adjacent to $\pm[i j k \ell]$ via the corresponding black triangle, and each of the octahedra $\mp[i j k M]$ and $\pm[i j \ell M]$ has to cancel away with the corresponding octahedron coming the 4D corner which is coming from the 3-cell adjacent to $\pm[i j k \ell]$ via the corresponding white triangle.

Assume that $X=x_{i j k}$. Then for each white tetrahedron $\pm\lceil i j k \ell\rceil \in \sigma$ we added the three octahedra $\pm T_{k}[i j \ell M], \mp T_{j}[i k \ell M]$, and $\pm T_{i}[j k \ell M]$ as well as the white tetrahedron $\mp\lceil i j k M\rceil$ to $\Sigma$ which do not belong to $\sigma$. Moreover, $\pm\lceil i j k \ell\rceil$ has three white triangular facets adjacent to $x_{i j k}$, namely $\mp T_{k}\lceil i j \ell\rceil$, which is the common triangle with $\pm T_{k}[i j \ell M], \pm T_{j}\lceil i k \ell\rceil$, which is the common triangle with $\mp T_{j}[i k \ell M]$, and $\mp T_{i}\lceil j k \ell\rceil$, which is the common triangle with $\pm T_{i}[j k \ell M]$. Therefore, each of these octahedra has to cancel away with the corresponding octahedron from the $4 \mathrm{D}$ corner which is coming from the 3 -cell adjacent to $\pm\lceil i j k \ell\rceil$ via the corresponding white triangle.

Consider two 3-cells $\Omega, \bar{\Omega} \in \sigma$ adjacent via the black triangle $\lfloor i j k\rfloor$, say $\lfloor i j k\rfloor$ belongs to $\Omega$ and $-\lfloor i j k\rfloor$ belongs to $\bar{\Omega}$. Then the 4D corner corresponding to $\Omega$ contributes the black tetrahedron $-\lfloor i j k M\rfloor$ to $\Sigma$, whereas the 4D corner corresponding to $\bar{\Omega}$ contributes the black tetrahedron $\lfloor i j k M\rfloor$ to $\Sigma$. Therefore, the latter two black tetrahedra cancel out.

Consider two 3-cells $\Omega, \bar{\Omega} \in \sigma$ adjacent via the white triangle $\lceil i j k\rceil$, say $\lceil i j k\rceil$ belongs to $\Omega$ and $-\lceil i j k\rceil$ belongs to $\bar{\Omega}$. Then the 4D corner corresponding to $\Omega$ contributes the octahedron $-[i j k M]$ to $\Sigma$, whereas the 4D corner corresponding to $\bar{\Omega}$ contributes the octahedron $[i j k M]$ to $\Sigma$. Therefore, the latter two octahedra cancel out.

Up to now we proved that all black tetrahedra and all octahedra in $\Sigma \backslash \sigma$ cancel out. We will now consider with the white tetrahedra in $\Sigma \backslash \sigma$.

Lemma 6.1 The white tetrahedra $\lfloor i j k M\rfloor$ arising in the third step of the algorithm build flowers which only contain white tetrahedra.

Proof We have two prove that each of these white tetrahedra has exactly three adjacent white tetrahedra in the flowers, one via each white triangle adjacent to $X$. They are not adjacent to the 3-cells in $\sigma$, but each of them has three common neighbors adjacent to $X$ with the corresponding white tetrahedron in $\sigma$. These common neighbors are octahedra which cancel out in the previous steps of the algorithm. 
Consider now two white tetrahedra $T, \bar{T} \in \Sigma \backslash \sigma$ after the third step of the algorithm, where the corresponding white tetrahedra in $\sigma$ are adjacent, i.e., there is a pair of octahedra with the same set of points and different orientation, one adjacent to $T$ and the other adjacent to $\bar{T}$. Therefore, $T$ and $\bar{T}$ share a common white triangle (up to orientation), i.e., they are adjacent.

Consider the 4D corner which we add to $\Sigma$ for an octahedron. Its two octahedra which do not belong to $\sigma$ share a common white triangle (up to orientation) which does no lie in $\sigma$. Furthermore, consider a sequence of adjacent octahedra in $\sigma$, where the common triangles are all white triangles. Then the octahedra in the corresponding 4D corners which are not in $\sigma$ all share a common white triangle (up to orientation).

Consider now two white tetrahedra $T, \bar{T} \in \Sigma \backslash \sigma$ after the third step of the algorithm, where the corresponding tetrahedra in $\sigma$ are connected by a sequence of octahedra adjacent via white triangles. Then, there is a pair of octahedra, one of them adjacent to $T$ and the other one adjacent to $\bar{T}$, which share a common white triangle (up to orientation). This triangle does not belong to any 3-cell in $\sigma$ and, therefore, is a common triangle of $T$ and $\bar{T}$, i.e., $T$ and $\bar{T}$ are adjacent.

Now we continue with the proof of Theorem 2.4. We already proved that a flower containing only black tetrahedra can be written as a sum of 3D corners on black 4simplices (see proof of step 1). Analogously, one can write every flower containing only white tetrahedra as a sum of 3D corners on white 4-simplices. So we write for each of the flowers of white tetrahedra in $\Sigma \backslash \sigma$ after the third step of the algorithm the flower of opposite orientation as a sum of 3D corners on white 4-simplices and add this sums to $\Sigma$. Then $\Sigma=\sigma$.

Open Access This chapter is distributed under the terms of the Creative Commons AttributionNoncommercial 2.5 License (http://creativecommons.org/licenses/by-nc/2.5/) which permits any noncommercial use, distribution, and reproduction in any medium, provided the original author(s) and source are credited.

The images or other third party material in this chapter are included in the work's Creative Commons license, unless indicated otherwise in the credit line; if such material is not included in the work's Creative Commons license and the respective action is not permitted by statutory regulation, users will need to obtain permission from the license holder to duplicate, adapt or reproduce the material.

\section{References}

1. Adler, V.E., Bobenko, A.I., Suris, Y.B.: Classification of integrable discrete equations of octahedron type. Intern. Math. Res. Not. 2012(8), 1822-1889 (2012)

2. Bobenko, A.I., Suris, Y.B.: Discrete pluriharmonic functions as solutions of linear pluriLagrangian systems. Commun. Math. Phys. 336(1), 199-215 (2015)

3. Boll, R., Petrera, M., Suris, Y.B.: Multi-time Lagrangian 1-forms for families of Bäcklund transformations. Toda-type systems. J. Phys. A: Math. Theor. 46(275204) (2013)

4. Boll, R., Petrera, M., Suris, Y.B.: What is integrability of discrete variational systems? Proc. R. Soc. A 470(20130550) (2014) 
5. Boll, R., Petrera, M., Suris, Y.B.: Multi-time Lagrangian 1-forms for families of Bäcklund transformations. Relativistic Toda-type systems. J. Phys. A: Math. Theor. 48(085203) (2015)

6. Boll, R., Petrera, M., Suris, Y.B.: On integrability of discrete variational systems. Octahedron relations. Intern. Math. Research Notices rnv140 (2015)

7. Conway, J.H., Sloane, N.J.A.: The cell structures of certain lattices. In: Hilton, P., Hirzebruch, F., Remmert, R. (eds.) Miscellanea Mathematica, pp. 71-107. Springer, Berlin (1991)

8. Doliwa, A.: Desargues maps and the Hirota-Miwa equation. Proc. R. Soc. A 466, 1177-1200 (2010)

9. Doliwa, A.: The affine Weyl group symmetry of Desargues maps and of the non-commutative Hirota-Miwa system. Phys. Lett. A 375, 1219-1224 (2011)

10. Lewin, L.: Polylogarithms and Associated Functions. Elsevier North Holland, New York (1981)

11. Lobb, S., Nijhoff, F.W.: Lagrangian multiforms and multidimensional consistency. J. Phys. A: Math. Theor. 42(454013) (2009)

12. Lobb, S., Nijhoff, F.W.: Lagrangian multiform structure for the lattice Gel'fand-Dikii hierarchy. J. Phys. A: Math. Theor. 43(072003) (2010)

13. Lobb, S., Nijhoff, F.W., Quispel, G.R.W.: Lagrangian multiform structure for the lattice KP system. J. Phys. A: Math. Theor. 42(472002) (2009)

14. Moody, R.V., Patera, J.: Voronoi and Delaunay cells of root lattices: classification of their facets by Coxeter-Dynkin diagrams. J. Phys. A: Math. Gen. 25(5089) (1992)

15. Suris, Y.B.: Variational formulation of commuting Hamiltonian flows: multi-Lagrangian 1forms. J. Geom. Mech. 5, 365-379 (2013)

16. Suris, Y.B.: Variational symmetries and pluri-Lagrangian systems. arXiv:1307.2639 [math-ph] (2013)

17. Yoo-Kong, S., Lobb, S., Nijhoff, F.W.: Discrete-time Calogero-Moser system and Lagrangian 1-form structure. J. Phys. A: Math. Theor. 44(365203) (2011) 